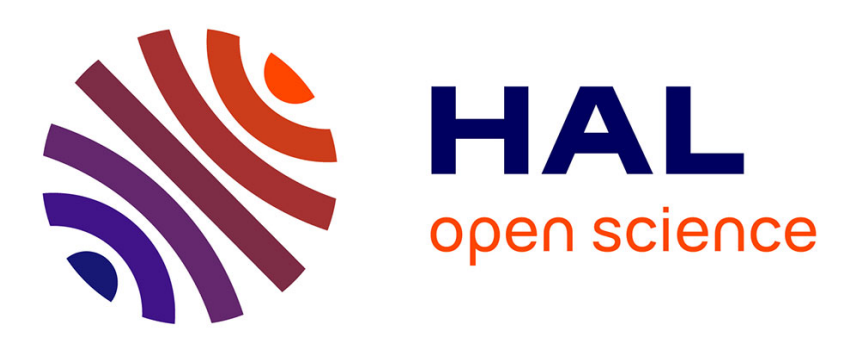

\title{
On the importance of the submicrovascular network in a computational model of tumour growth.
}

Anne-Cécile Lesart, Boudewijn van Der Sanden, Lauriane Hamard, François Estève, Angélique Stéphanou

\section{To cite this version:}

Anne-Cécile Lesart, Boudewijn van Der Sanden, Lauriane Hamard, François Estève, Angélique Stéphanou. On the importance of the submicrovascular network in a computational model of tumour growth.. Microvascular Research, 2012, 84 (2), pp.188-204. 10.1016/j.mvr.2012.06.001 . inserm00738177

\section{HAL Id: inserm-00738177 https://www.hal.inserm.fr/inserm-00738177}

Submitted on 3 Oct 2012

HAL is a multi-disciplinary open access archive for the deposit and dissemination of scientific research documents, whether they are published or not. The documents may come from teaching and research institutions in France or abroad, or from public or private research centers.
L'archive ouverte pluridisciplinaire HAL, est destinée au dépôt et à la diffusion de documents scientifiques de niveau recherche, publiés ou non, émanant des établissements d'enseignement et de recherche français ou étrangers, des laboratoires publics ou privés. 


\title{
On the Importance of the Submicrovascular Network in a Computational Model of Tumour Growth
}

\author{
Anne-Cécile Lesart ${ }^{\mathrm{a}}$, Boudewijn van der Sanden ${ }^{\mathrm{b}}$, Lauriane Hamard ${ }^{\mathrm{b}}$, François Estève ${ }^{\mathrm{c}}$, Angélique \\ Stéphanou ${ }^{\mathrm{a}, 1, *}$ \\ ${ }^{a}$ UJF-Grenoble 1, CNRS, Laboratory TIMC-IMAG UMR 5525, DyCTiM research team, \\ Grenoble, F-38041, France \\ ${ }^{b}$ Grenoble Institut des Neurosciences - GIN, INSERM U836, Equipe 7, \\ Chemin Fortuné Ferrini, BP 170, 38042 Grenoble Cedex 9, France. \\ ${ }^{c}$ Grenoble Institut des Neurosciences - GIN, INSERM U836, Equipe 6, \\ Chemin Fortuné Ferrini, BP 170, 38042 Grenoble Cedex 9, France.
}

\begin{abstract}
A computational model is potentially a powerful tool to apprehend complex phenomena like solid tumour growth and to predict the outcome of therapies. To that end, the confrontation of the model with experiments is essential to validate this tool. In this study, we develop a computational model specifically dedicated to the interpretation of tumour growth as observed in a dorsal skinfold chamber on a mouse. Observation of the skin vasculature at the dorsal window scale shows a sparse network of a few main vessels of several hundreds micrometers in diameter. However observation at a smaller scale reveals the presence of a dense and regular interconnected network of capillaries about ten times smaller. We conveniently designate this structure as the submicrovascular network (SMVN) ${ }^{2}$, although improperly considering the scale. The question that we wish to answer concerns the necessity of explicitly taking into account the SMVN in the computational model to describe the tumour evolution observed in the dorsal chamber. For that, simulations of tumour growth realised with and without the SMVN are compared and lead to two distinct scenarios. Parameters that are known to strongly influence the tumour evolution are then tested in the two cases to determine to which extent those parameters can be used to compensate the observed differences between these scenarios. Explicit modelling of the smallest vessels appears mandatory although not necessarily under the form of a regular grid. A compromise between the two investigated cases can thus be reached.

Keywords: computational model, dorsal skinfold chamber, microvascular network, angiogenesis, tumour growth, multiscale model, hybrid model
\end{abstract}

\footnotetext{
${ }^{*}$ Corresponding author

Email address: Angelique.Stephanou@imag.fr (Angélique Stéphanou)

${ }^{1}$ Tel: +330456520041 / Fax: +330456520022

${ }^{2}$ SMVN: Sub-Microvascular Network
} 


\section{Introduction}

Tumour growth is a complex phenomena which is regulated by different processes. An integrated view of these processes which interact on multiple scales is therefore necessary in order to understand and apprehend them. Theoretical models are useful tools in order to reach a more complete understanding of the phenomena by reproducing how this biological system works. At the end of the sixties, the first models on tumour growth already described how the structure and the growth of multicellular spheroids could be affected by the environmental conditions in oxygen and nutrients (Burton, 1966; Greenspan, 1972). Since then, mathematical models have become more and more complex. In particular, in the eighties, angiogenesis began to be considered explicitly to model oxygen and nutrients availability on the tumour. Vascular growth was first modelled in one dimension (Balding and McElwain, 1985). Other works followed to extend the model in two or three dimensions (Anderson and Chaplain, 1998; Stéphanou et al., 2005; Stokes and Lauffenburger, 1991). This change of dimension permits to take into account the spatial distribution of the vascular network. Hybrid models combining different modelling approaches then emerged to model the different processes interacting on tumour growth (Alarcòn et al., 2003; Anderson and Chaplain, 1998; Perfahl et al., 2011; Stokes and Lauffenburger, 1991; Welter et al., 2008). Discrete models such as cellular automata are found to be particularly adequate to model the growth of small spheroids because they allow us to consider intrinsic parameters for each cells such as its age in the cell cycle. They are often coupled with continuous equations which describe vascular remodelling, blood flow, oxygen and nutrients diffusion (Alarcòn et al., 2003; Welter et al., 2008). Interested readers can refer to the recent reviews by Tracqui (2009) and Byrne (2010) on the evolution of the theoretical models on tumour growth over the last decades.

The current trend in theoretical modelling is to closely link, on a quantitative basis, theoretical and experimental models. The aim is to produce computational tools as close as possible to reality. In the case of cancer, most of the models coupling theory and experiments aim at testing therapies (Frieboes et al., 2009; Gatenby et al., 2009). Our work follows this trend. Our goal is to develop a computational tool to describe the tumour evolution as it is observed on a dorsal skinfold chamber on a mouse. This technique is known for several years (Asaishi et al., 1981; Lehr et al., 1993) and provides a mean to observe accurately the development of the tumour over time. Moreover, intravital microscopy permits to visualise as well the evolution of rheological and functional parameters such as blood flow and vascular density. Thus, the use of FITC-Dextran, a fluorescent agent, provides a mean to observe angiogenesis through a bi-photonic microscope (Dellian et al., 1996; Koehl et al., 2009; Strieth et al., 2004). Similarly, rhodamine highlights blood cells so as to determine blood flow and vessels permeability (Fukumura and Jain, 2008; 
Strieth et al., 2008). Oxygen concentration in the tissue can also be measured with the use of oxygen sensors (Koehl et al., 2009). The dorsal chamber method was already used to validate theoretical models that describe the vascular permeability for oxygen (Dedeugd et al., 2009; Strieth et al., 2008). However, this technique is not used yet to validate a complex model of tumour growth.

The resolution of the current microscopes and the use of fluorescent probes such as FITC-dextran allow us to visualise a hierarchy in the vascular network. Arterioles and venules can be distinguished with typical radius a few tens of micrometers. Smaller vessels (capillaries) with radius under 10 microns are visible with a higher resolution. We define the "sub-microsvascular network" (SMVN) as this regular pre-existing and healthy microvascular network of capillaries that ensures physiological oxygenation of the tissue. Some models do not take into account the SMVN to describe tumour growth (Chaplain et al., 2006; McDougall et al., 2006; Owen et al., 2009; Stéphanou et al., 2005). However, in more recent models, it is considered that the evolution of the SMVN is necessary to describe it accurately (Bartha and Rieger, 2006; Lee et al., 2006; Paul, 2009; Shirinifard et al., 2009; Welter et al., 2008; Welter and Rieger, 2010). It was shown that major disruptions could occur on these small microvessels during tumour growth and influence the process. The main question in this paper is to determine if it is necessary to explicitly describe this SMVN in the tissue skin of the mouse to reproduce tumour growth as observed with the dorsal skinfold chamber.

To that goal, we developed a hybrid model inspired from several existing ones. Tumour growth is based on the cellular automaton proposed by Alarcòn et al. (2003). This choice for an automaton allows us to describe the tumour cells transitions between different states (proliferative, quiescent, apoptotic and necrotic) depending on the environmental constraints, mainly space and oxygen availability. Moreover, this model takes into account the cell cycle by assigning a timer to each individual cell. The cell sensitivity to hypoxia, which depends on the phase of the cell cycle, can be finely expressed. Angiogenesis and blood flow in the microvasculature are directly based on the model by Stéphanou et al. (2006). The new vessels emerging from the existing ones proliferate and migrate in response to combined chemotactic and haptotactic cues of growth factors (from hypoxic tumour cells) and matrix fibres respectively. The distribution of red blood cells in the vascular network allows us to estimate the oxygen field in the tissue based on vessel permeability for oxygen, and oxygen diffusion and consumption. Finally, conditions for vessels sprouting and vessels collapsing have been introduced, both based on the hypotheses proposed by Welter and Rieger (2010). 
Although vascular recruitment and vessels adaptation are clearly visible in biological experiments, it remains difficult to determine if the neovasculature emerges from the main vessels or from the smaller ones forming the SMVN. In order to answer this question, the numerical simulations presented in this paper compare tumour growth with and without the SMVN. Those simulations are based upon experimentally observed initial networks of main vessels to reproduce the initial experimental conditions. The impact of the SMVN on the evolution of the tumour cells quantity and on the tumour expansion rate is evaluated.

Parameters which strongly influence the tumour evolution are then specifically considered. First of all, the tumour cells resistance to hypoxia is evaluated by changing the sensitivity of the cells to the lack of oxygen. This can be related to different cell phenotypes. The effect of the sprouting ability of the vascular network is also evaluated to determine whether an increased angiogenesis could compensate the initially reduced density when the SMVN is not considered.

\section{Experimental background}

Experiments realised with the dorsal skinfold chamber on the mouse allow to observe with many details the process of angiogenesis and tumour growth. The tumour under the form of isolated cells, spheroid, or tumour cells embedded in a piece of matrigel is grafted at the surface of the skin under the observation window. Under such conditions, tumour cells rapidly seek to recruit a vascular network by releasing a diversity of growth factors among which the vascular endothelial growth factor (VEGF). In less than two days, the angiogenic response is visible. This starts with the apparition of bleeding spots. The vessels become leaky as the endothelial cells forming the vessel walls start to respond to the VEGF signals. They do so by releasing the bonds between them and by migrating up the source of the signal. This event is shortly followed by vascular remodelling and vessels dilation.

In 3 days the tumour implant is surrounded by a diffuse mass of small vessels (Fig. 1). Vessels which initially formed inside the tumour mass are often crushed by the increased pressure from the proliferating cells. Very few vessels can subsequently penetrate the tumour to deliver the oxygen. This leads to the formation of the necrotic core in the most central part, surrounded by a thin rim of quiescent cells under the hypoxic stress. Only the cells located at the most peripheral part of the tumour, where space and oxygen are still available, remain able to proliferate.

Macrofluorescence imaging, involving the use of fluorescent probes (FITC-dextran, rhodamine) into the vascular system of the mouse, gives access to very detailed information about the structure of the 
neovasculature, the red blood cells distribution into the network and the vessels permeability. The use of fluorescent tumour cells, obtained by transfection, provides a more quantitative information about cell proliferation, death and invasion. The quantification possibilities offered by the dorsal chamber setup are huge. In this paper we will only remain on a qualitative basis as a first step. The progressive integration of these new elements is envisaged in a near future.

Note: details on the experimental procedure about the dorsal skinfold chamber that we used, can be found in Maurin et al. (2011).

\section{Skeletonization of the vascular network}

All procedures described for image analysis are performed using ImageJC software.

First, images are converted into grey levels. Then a window of interest is selected and is chosen to correspond to a $5.5 \mathrm{~mm}$-long square. This window is discretized by a $220 \times 220$ grid, where each element is a square of $25 \mu \mathrm{m}$ which corresponds to the size of a single cell. A main traversing vessel (across the window) is arbitrarily selected. One of its extremities is taken as the reference point (big grey bullet mark in figure 2) from which the parameters defining the first vessel segment are measured. Each segment $S_{i}$ is defined by 3 parameters which are its length $L_{i}$, its diameter $D_{i}$ and its inclination $I_{i}$ from the horizontal. The segments are numbered successively according to the following rules:

1. each time a bifurcation is encountered, the right segment is numbered first until the end of the branch (a change in vessel inclination bigger than 5 degrees leads to a new segment),

2. we then return to the last (closest) bifurcation encountered and repeat successive numbering of the right segments along the branch and so on, until all branches are segmented.

\section{Computational model}

The computational model is developed in order to simulate experimental observations made from the dorsal skinfold chamber. It aims at describing the successive phases of tumour evolution starting with (i) the initial avascular growth phase, followed by (ii) the hypoxic switch for tumour cells leading to growth factor diffusion, (iii) the resulting angiogenic phase and (iv) the ultimate phase of vascular-depending tumour growth. It is based on the coupling between two main modules under the form of a cellular automaton to properly describe events down to the cell scale. The first module describes the tumour evolution and integrates the cell cycle and metabolism (oxygen consumption, hypoxia, cell proliferation and cell death) to simulate the hypoxic switch and the release of growth factor. The second module 
describes the angiogenic process by which the tumour becomes vascularised under the effect of the growth factor. Angiogenesis involves vessels sprouting, branching and collapsing in response to blood rheological constraints. These two modules are presented in detail in the following sections and relations between them are summarized in the following diagram (Fig. 3).

\section{Simulation domain}

The dorsal skinfold chamber comprises a titanium frame on one side of the skinfold and a glass cover on the other side. The tumour implanted is therefore sandwiched between the frame and cover and constrained to grow mainly in a 2D space. Since events in the third dimension are minimal, the computational model is based on a cellular automaton evolving on a 2D grid. The simulation domain (Fig. 4) is a $5.5 \mathrm{~mm}$-long square, discretized by a regular $(i, j)$-grid $(G)$ with $220 \times 220$ elements. The resulting size of each element is $\Delta x=\Delta y=25 \mu \mathrm{m}$ so as to correspond to the approximate size of a real cell. A tumour cell thus occupies an element $e(i, j)$ of the grid and a vessel segment is defined between two horizontal or vertical nodes $N(i, j)$ of this grid.

\section{Tumour growth}

The tumour evolution is modelled by a cellular automaton inspired from the one previously described in Alarcòn et al. (2003). It is defined on the grid $G$ where each element $e$ can be empty $(e=0)$ or contain a tumour cell which can either be proliferative, quiescent, apoptotic or necrotic ( $e=1,2,3$ or 4 respectively).

The transitions between the different cell types are conditioned by the cell cycle and environmental conditions. More specifically the level of oxygen available in the tissue and the available space will determine if the cell will proliferate or die. The rules presented in the flowchart (Fig. 5) are as follows:

- a proliferative cell becomes quiescent if the level of oxygen is below the admissible threshold for proliferation $\alpha_{p}$, the cell should additionally be phase-sensitive to hypoxia, that is in phase $G_{1}$. The duration of this phase is given by $t_{G_{1}}$.

- if the level of oxygen is above the admissible threshold $\alpha_{p}$, then the cell can divide in two daughter cells, if in phase $M$, provided there is enough available space. This means that an empty elements $e=0$ adjacent to the cell must exist. This element receives one of the daughter cell, whereas the other daughter cell replaces the mother cell. The cell cycle duration is ruled by the parameter $t_{c y c l e}$. 
- if there is not enough space then the initial proliferative cell enters apoptosis. The apoptotic stage lasts for a time $t_{\text {apop }}$ during which the cell keeps occupying the grid element until the cell is removed which corresponds to the release of the grid element.

- a quiescent cell can come back to a proliferative state if the level of oxygen becomes normal again $\left(\geq \alpha_{p}\right)$. However, if the oxygen level keeps decreasing below the admissible quiescence threshold $\alpha_{q}$ and if the cell is exposed for too long to this increased stress (for longer than time $T_{\text {limit }}$ ) then the cell becomes necrotic. The value of the quiescent threshold $\alpha_{q}$ reflects the cell resistance to hypoxia. In the case of necrotic death, the grid element occupied by the cell is never released.

Note: according to the rule of the cellular automaton, it is assumed that only the $G_{1}$ phase is sensitive to hypoxia. This means that if a cell exposed to hypoxia while in another phase of its cycle $\left(S, G_{2}\right.$ or $M)$ it will pursue its cycle unaffected by the lack of oxygen. However if there is enough space for the cell to divide, the daughter cells which start their cycles in $G_{1}$ will immediately enter the quiescent state. This assumption can be justified by the fact that the $G_{1}$ phase is the longest and most energy consuming since. It is the phase during which the cell synthesizes the proteins, including the enzymes, required for the subsequent phases. It is consequently assumed that once the cell has passed the $G_{1}$ phase, it has accumulated enough energy to complete mitosis and oxygen is no longer necessary.

\section{Cell division}

The cell can divide if there is an empty element $e$ adjacent to its position among the 8 adjacent elements. It is assumed that one of the daughter cell takes place in this element whereas the other daughter cell replaces the mother cell. The probability for the daughter cell to occupy a specific empty adjacent position is proportional to the distance between this position and the mother cell position. So, the probability for a daughter cell to fill one of the 4 transverse elements (right, left, up or down) is denoted $p$ and the probability to occupy one of the 4 diagonal elements is $p / \sqrt{2}$. The probabilities are calculated so that the sum of probabilities for the adjacent cells surrounding one dividing cell is equal to one.

\section{Angiogenesis}

The angiogenic process starts when tumour cells are lacking oxygen and nutrients to sustain their growth. The cells then drastically reduce their metabolism by stopping proliferation and thus reducing their need for oxygen. This is the quiescent state or $G_{0}$-phase which can last for several months depending on the tumour cell type. 
In this state, the cells seek to recruit a neovasculature to bring back the oxygen that they need to return to proliferation. To that aim the quiescent cells massively release a vascular endothelial growth factors (VEGF) which induces angiogenesis from the neighbouring vessels. The endothelial cells forming the vessel walls detached from one another and migrate through the tissue fibres towards the source of growth factor which also stimulate their proliferation. Some sprouts progressively emerge from the parent vessels and grow guided by the VEGF-gradient. Growing vessels can form branches and connect to other growing vessels to form some loops by anastomosis. In this angiogenic process, the endothelial cells produce matrix metalloproteinase (MMPs) that allow them to degrade the surrounding matrix of fibres (mainly fibronectin FN) and progress through the tissue.

\section{VEGF evolution}

The variable $V(x, y, t)$ denotes the VEGF concentration at location $(x, y)$ and time $t$ and $\tilde{V}(e)$ denotes its production rate at each grid element $e$. VEGF is assumed to be emitted with a constant rate $\tilde{V}_{\text {emit }}$ by quiescent cells only $(e=2)$, so $\tilde{V}(2)=\tilde{V}_{\text {emit }}$ and $\tilde{V}(e \neq 2)=0$. The VEGF evolution is then described by the following partial differential equation inspired and adapted from Bauer et al. (2007):

$$
\frac{\partial V}{\partial t}=\tilde{V}(e)+D_{V} \nabla^{2} V-\lambda V-\eta \tilde{V}_{\text {uptake }}
$$

where $D_{V}$ is the VEGF diffusion coefficient, $\lambda$ the VEGF decay rate and $\eta$ the uptake rate of VEGF by endothelial cells. As in Bauer et al. (2007), $\tilde{V}_{\text {uptake }}$ depends on the local amount of available VEGF, $V(e)$ and this uptake cannot exceed a maximum value $V_{\max }$ corresponding to the saturation of all the VEGF receptors. For each element $e$ of the grid, the VEGF consumption of the $n$ blood vessels $v$ adjacent to $e$ are added $\left(n_{\max }=4\right)$. $\tilde{V}_{\text {uptake }}$ is thus evaluated from:

$$
\tilde{V}_{\text {uptake }}(e)=\sum_{v=1}^{n} \min \left(V(e), V_{\max }\right)
$$

Vessels growth

Endothelial cells forming the parent vessels walls start to detach, proliferate and migrate to form some sprouts under the effect of VEGF. The cells then migrate up the VEGF gradient (chemotaxis), while taking support on the fibres of the extracellular matrix (haptotaxis). The mathematical model initially proposed in Anderson and Chaplain (1998) to describe this scenario using a Hybrid Discrete Continuum (HDC) formulation has been since extensively used with some variations or additions on these main hypotheses (Anderson and Chaplain, 1998; Plank et al., 2004; McDougall et al., 2006; Stéphanou et al., 
2006; Macklin et al., 2009). The principle of the HDC model is to couple continuum equations with a cellular automaton so as to be able to describe the individual and specific characters of the cells. This provides a finer description of localised events of cell-cell interaction and cell migration (full details of the HDC model as it is implemented here can be found in Stéphanou et al. (2006)). With such a description, the displacement of the endothelial cells $n_{i, j}$ in the tissue, discretised using the 2D-grid (Fig. 4), is ruled by a set of probabilities conditioned by the local environmental conditions as follows:

$$
n_{i, j}^{q+1}=n_{i, j}^{q} P_{0}+n_{i+1, j}^{q} P_{1}+n_{i-1, j}^{q} P_{2}+n_{i, j+1}^{q} P_{3}+n_{i, j-1}^{q} P_{4}
$$

where the position of the cell $n_{i, j}$ at discrete time $q+1$ depends on the probabilities $P_{0}$ to $P_{4}$ for the cell at previous time $q$ to remain where it stands or to move in one of the four directions of the 2D-grid (right, left, down or up respectively). These probabilities are related to the local gradients of VEGF denoted $V(x, y, t)$ and matrix fibres (such as fibronectin) denoted $f(x, y, t)$. VEGF is produced by the hypoxic tumour cells and its evolution is given by equation 1 . The matrix fibres evolution is given by the following set of partial differential equations:

$$
\begin{aligned}
\frac{\partial f}{\partial t} & =\beta n_{i, j}-\gamma m f, \\
\frac{\partial m}{\partial t} & =\alpha n_{i, j}+D \nabla^{2} m-\nu m,
\end{aligned}
$$

where $\beta$ is the production rate of fibres by one endothelial tip cell of a sprout $n_{i, j}\left(n_{i, j}\right.$ is a boolean variable with value 1 or 0 , to materialize the existence or absence of a sprout tip at the corresponding grid node respectively). Endothelial cells simultaneously produce, with rate $\alpha$, a matrix degrading enzyme $m(x, y, t)$ (metalloproteinase) to ease the migration through the tissue. The matrix is degraded by the enzyme with rate $\gamma$. The enzyme diffuses in the tissue with diffusion coefficient $D$ and decays with rate $\nu$ (details on this model can be found in Stéphanou et al. (2006) and McDougall et al. (2010)). The initial distribution of matrix fibres forming the tissue, that is before angiogenesis starts, is assumed to be homogeneous with concentration value $f(x, y, 0)=f_{0}$.

\section{Vessels sprouting}

As in the reference model (Stéphanou et al., 2006), we assume that the sprouting ability of the vessels increases with the local VEGF concentration (Qutub and Popel, 2009; Welter et al., 2009). We further assume as in Welter et al. (2008) that a forming sprout inhibits further sprouting of the vessel. We set this inhibition distance to $50 \mu \mathrm{m}$. Vessels sprouting inside the tumour is different than in the normal tissue. Indeed, the tumour changes the nature of the vessel wall by stopping angiogenic sprouting after a

time delay $t_{E C}^{(\text {switch) }}$ following the growth of the tumour over the considered vessel (Welter et al., 2009). 
Vessels collapse

Inside the tumour, vessels are unable to recruit pericytes and vessels are much more unstable. In particular, when the wall shear stress $\tau_{w}$ is too low vessels tend to collapse. In our computational model, we assume that if the wall shear stress falls below a threshold value $\tau^{\text {coll }}$, that is $\tau_{w}<\tau^{\text {coll }}$ then the vessel has a mean survival time $t_{E C}^{(\text {death) }}$ (Welter et al., 2009). The expression of $\tau_{w}$ is given next.

\section{Oxygen diffusion}

The oxygen distribution in the tumour strongly impacts on the tumour cells by affecting tumour cells states (proliferative, quiescent, necrotic). Oxygen molecules are transported by the red blood cells (RBCs) through the vessels. These cells can only circulate in vessels with a radius $\geq 4 \mu \mathrm{m}$. From the hematocrit, which is the proportion of blood volume occupied by circulating cells in the plasma (where the RBCs represent the biggest fraction), the amount of oxygen in the tissue can be evaluated.

\section{Blood transport in vessels}

RBCs in each vessel section of the network are transported at the speed of the blood flow $Q$. RBCs reaching each node of the vascular network are distributed in the vessels with outgoing amount proportional to the blood flow in each vessel. The blood flow obeys the Poiseuille's law. It depends on the vessel radius $R$ and length $L$. It also linearly depends on the pressure drop $\Delta P$ between the vessels extremities and on the blood viscosity. The blood being a bi-phasic medium with a fluid phase for the plasma and a solid phase comprising the red blood cells, its viscosity $\mu$ is not constant and varies with the hematocrit $\left(H_{D}\right)$ and with the radius $(R)$ of the vessel where it flows. The resulting blood flow in each vessel is thus given by:

$$
Q=\frac{\pi R^{4}}{8 L} \frac{\Delta P}{\mu\left(H_{D}, R\right)}
$$

The function $\mu\left(H_{D}, R\right)$ used to describe the apparent viscous behaviour of the blood has been derived from experimental measurements (Pries et al., 1994). The interested reader is referred to this study for details. This function has been extensively used in mathematical models and is also detailed in the following contributions (Alarcòn et al., 2003; Stéphanou et al., 2006; McDougall et al., 2006; Owen et al., 2009; Wu et al., 2009; McDougall et al., 2010).

Blood flow is then computed on the basis of mass conservation at each nodes of the network. This means that the sum of the flows at each node $(i, j)$ of the $2 \mathrm{D}$-grid equals to zero. This procedure leads to a set of linear equations for the nodal pressures $(P)$ which is numerically solved using a Successive 
Over-Relaxation (SOR) method. Once nodal pressures are computed, the flows in each vessels can be deduced from equation (6). The wall shear stress can easily be calculated from the blood flow and is used to determine which vessels will collapse in the tumour.

$$
\tau_{w}=\frac{4 \mu\left(H_{D}, R\right)}{\pi R^{3}}|Q|
$$

Oxygen transport from the vessels to the tissue

Oxygen diffuses through the vessels walls and into the tissue where it is consumed by the tissue cells. The spatial profile of oxygen concentration is given from the following partial differential equation:

$$
\frac{\partial C}{\partial t}=D_{O_{2}} \nabla^{2} C+\tilde{C}_{\text {emit }}-\tilde{C}_{\text {cons }}
$$

where $C$ is the concentration of oxygen in the tissue, $\tilde{C}_{e m i t}$ is the rate of oxygen passing through the vessels walls (emission rate of oxygen into the tissue) and $\tilde{C}_{c o n s}$ is the rate of oxygen consumed by the tissue cells where each tissue cell occupies one element $e$ of the grid in the discrete model (Fig. 4). $\tilde{C}_{e m i t}$ depends on the concentration difference of oxygen between the interior of the vessel $H_{v}$ (assumed proportional to the hematocrit) and the exterior $C(e)$ (Alarcòn et al., 2003). It also depends on the permeability of the vessel walls $\gamma_{p}$. For each element $e$ of the simulation grid, the oxygen contribution of the $n$ blood vessels $v$ adjacent to $e$ are added $\left(n_{\max }=4\right) . \tilde{C}_{e m i t}(e)$ is thus evaluated from:

$$
\tilde{C}_{e m i t}(e)=\gamma_{p} \sum_{v=1}^{n}\left(H_{v}-C(e)\right)
$$

Although the oxygen tends to decrease as it is distributed throughout the network, we here consider that this decrease is negligible. It is therefore assumed that the total amount of oxygen carried through the vessels is not limiting. The consumption rate of oxygen by the tissue cells depends on the type of cells and is linearly dependent upon the oxygen concentration $C$ similarly to Alarcòn et al. (2003):

$$
\tilde{C}_{\text {cons }}= \begin{cases}k_{N} C & \text { for normal cells } \\ k_{P} C & \text { for tumour proliferative cells } \\ k_{Q} C & \text { for tumour quiescent cells } \\ 0 & \text { for tumour necrotic and apoptotic cells }\end{cases}
$$

with $k_{P}>k_{N}>k_{Q}$. 


\section{Results}

Initial conditions

The microvascular network is extracted from experimental images acquired through the dorsal skinfold chamber. Pressures are then defined for each extremity of the vessels. The biggest pressure drops are assigned to the main vessels. The radius of the initial tumour is $100 \mu \mathrm{m}$. The tumour is positioned at the same location than in experiments. It is assumed that all tumour cells are proliferative with a random age in the cell cycle from 0 to 24 hours. VEGF is assumed to be produced only by quiescent cells. Consequently, the initial VEGF concentration is set to 0 . The density of matrix fibres in the tissue is initially homogeneous. The initial oxygen concentration in the tissue is derived from the hematocrit, that is the rate of red blood cells, that we imposed in the main vessels. We set its value to 0.45 which corresponds to the normal physiological condition. The oxygen concentration field in the tissue evidently depends if the submicrovascular network (SMVN) is taken into account or not. The SMVN is defined as the regular pre-existing and healthy microvascular network that ensures physiological oxygenation of the tissue. Experimental observations with higher magnification reveals its presence (Fig. 6). It appears as a regular network of small vessels with approximate diameters of $8 \mu \mathrm{m}$. The interdistance measured between the vessels in a same plane is around $100 \mu \mathrm{m}$. This corresponds to data from the literature (Welter et al., 2008, 2009; Secomb et al., 2004). Oxygen diffusion parameters are thus set so that the oxygen provided by the vessels diffuse with a limited distance of $100 \mu m$ around them. We will consider two cases in the simulations, one without the SMVN, related to a weakly oxygenated tissue, and the other one integrating this SMVN (in addition to the microvascular network extracted from the experimental images) and related to a homogeneously oxygenated tissue.

All parameter values taken for the simulations are given in Tables c and c.

In the following, we first start by presenting numerical simulations which compare tumour growth with and without the SMVN, in order to determine the impact of the SMVN on tumour evolution. These simulations are based on the experimentally observed initial networks of main vessels presented in figure 1. We then have a closer look on the parameters that strongly impact on the tumour evolution. They are specifically considered in order to evaluate their action on tumour growth, so that the model can be adapted to a specific tumour in the future. 


\section{Influence of the SMVN on tumour growth}

\section{Weakly vascularised tissue}

Figure 7 presents the simulation of tumour growth where only the main vessels, extracted from the experimental image, are taken into account. Angiogenesis and tumour cell states (Fig. 7, line 1), oxygen concentration (Fig. 7, line 2), matrix fibres density (Fig. 7, line 3) and VEGF concentration (Fig. 7, line 4) are followed over 70 days.

After 10 days, we observe that the tumour is already vascularised. Since the tissue is initially poorly oxygenated, proliferative tumour cells rapidly become quiescent inducing VEGF release. The main vessels located nearer to the tumour are hit first by the VEGF and start sprouting and degrading the matrix fibre in the migration process to rapidly reach the tumour.

After 30 days, a necrotic core is visible surrounded by quiescent and proliferative cells which actively contribute in oxygen depletion. Some vessels perfusing the tumour mass collapse if blood perfusion (wall shear stress) is too low. This leads to the apparition of isolated necrotic clusters and heterogeneous oxygen distribution in the tumour mass visible 70 days later.

\section{Homogeneously vascularised tissue}

The SMVN is introduced in the simulation as the regular grid with the properties defined in the initial conditions. As previously, angiogenesis and tumour cell states (Fig. 8, line 1), oxygen concentration (Fig. 8, line 2), matrix fibres density (Fig. 8, line 3) and VEGF concentration (Fig. 8, line 4) are followed over 70 days.

The presence of the SMVN allows the tissue to be homogeneously oxygenated. Consequently the proliferative tumour cells introduced do not immediately become quiescent as in the previous case, but can duplicate. However, the small tumour mass, as it starts to grow during the first few days, induces the rapid collapse of the sub-microvascular vessels which are located where the tumour is developing. The first quiescent cells appear and produce the VEGF signal which promotes angiogenesis. The new vessels germinate from the SMVN first. An angiogenic ring spreads with time as the tumour grows thus maintaining a uniform oxygen field around the tumour. A thin proliferative ring of tumour cells at the periphery is enough to ensure the tumour growth. Quiescent cells form a second wider ring located beneath the proliferative one and ensure the steady diffusion of VEGF. A large necrotic core is visible at 
the centre of the tumour after 30 days. This necrotic core represents the biggest part of the tumour mass after 70 days. Matrix fibres degradation is due to the endothelial cells as they migrate to form new vessels. We note that the degradation pattern gives an indication on the speed of the angiogenic process since higher degradation corresponds to slow migrating cells which have more the time to degrade more deeply the matrix.

\section{Comparison of the two cases}

Figures 7 and 8 put in evidence qualitative differences on tumour growth without and with the SMVN. We now study these difference more quantitatively with comparisons of (i) the tumour growth rate, (ii) the tumour cell states evolution and (iii) the evolution of the VEGF concentration.

\section{Tumour growth rate}

Figures $9 \mathrm{a}$ and $9 \mathrm{~b}$ compare respectively the evolutions with time of the tumour cells quantities and of the tumour radius for the two tissue cases (i.e. with or without SMVN).

The growth of the tumour is quadratic (Fig. 9a). It is faster when the SMVN is considered with $40 \%$ more tumour cells after 70 days. The growth rates measured from the expansion of the tumour radius plotted in figure 9b gives $120 \mu \mathrm{m} /$ day for the case without SMVN and $140 \mu \mathrm{m} /$ day for the case with SMVN. The faster growth is directly due to the presence of oxygen delivered by the SMVN which maintains steady cells proliferation.

\section{Tumour cell states evolution}

Figure 10 compares the evolutions with time of the quantities of the tumour cells for each cell state and for each tissue case, without SMVN (Fig. 10a) and with SMVN (Fig. 10b).

In the case without SMVN (Fig. 10a), the population of proliferative cells dominates over the other cell types. After 70 days, there are three times more proliferative cells than quiescent or necrotic cells. The proliferative cell quantity increases proportionally to the surface (quadratically) contrary to the other cell types which increase proportionally to the radius (linearly).

In the case with SMVN (Fig. 10b), the appearance of quiescent and necrotic cells are delayed since it takes about a week for the proliferative cells to deplete oxygen and become quiescent and another week for the quiescent cells to die through necrosis due to severe or prolonged hypoxia. The amount of necrotic 
cells rapidly takes over after 30 days. Contrary to the previous case (without SMVN) the quantity of proliferative cells evolves linearly whereas the quantity of necrotic cells evolves quadratically to become three times higher than the proliferative or the quiescent cells quantity after 70 days.

\section{Evolution of the VEGF concentration}

VEGF is released by hypoxic cells to recruit a vascular network which will provide the missing oxygen. In this model we assumed that only quiescent tumour cells release VEGF.

The evolution of the total VEGF quantity in the simulation domain (Fig. 11b) thus follows the evolution of the quiescent cells population (Fig. 11a). These evolutions are compared for the two tissue cases, that is with or without SMVN. The evolution of the VEGF quantity is smoother than the evolution of the quiescent cells (Fig. 11b). The difference is due to VEGF spontaneous degradation and to its consumption by endothelial cells with a maximum uptake limited to $V_{\max }$.

Figure 11c shows the VEGF evolution between two consecutive days, in other words the daily VEGF evolution. VEGF consumption can be higher than its production. However, this is observed only for the tissue case without SMVN. This event is correlated with decreasing phases of the quiescent cells population (Fig. 11a).

Figure 11d shows the evolution of the maximum VEGF quantity in the tissue. Two very different evolution profiles are observed for the two tissue cases. In the case with SMVN, the maximum VEGF quantity increases rapidly to reach a plateau in 20 days. This is due to the homogeneous ring shape distribution of the quiescent cells. At the opposite, in the case without SMVN, the quiescent cells are inhomogeneously localised and form isolated clusters induced by the collapse of vessels in the tumour. These clusters produce outbursts of VEGF which explain the perturbed evolution of the maximum VEGF quantity. We note that one outburst of VEGF occurs a time $t=48$ days in the tissue case with SMVN. It is due to the collapse of one of the main vessel of the vasculature (Fig. 8d) which led to the sudden decrease of oxygen.

The VEGF evolution is thus much more steady with the SMVN. The effects of local "accidents", such as the local disruption of a main vessel, are quickly absorbed by the presence of the SMVN which tends to homogenize the oxygen conditions. At the opposite, they are amplified when this SMVN is absent. 


\section{Impact of some key parameters on tumour growth}

The parameters taken to run the simulations are not specific of a particular tumour type. Among the parameters that defined a tumour type, there is the tumour cells ability to resist to the lack of oxygen. In this section, the impact of this key parameter for the tumour evolution is evaluated so as to explore a broader spectrum of tumour growth behaviours that can be encountered. This will be useful to adapt the model to a specific tumour type at a later stage.

We finally evaluate a parameter related to the vasculature with its sprouting ability. The idea is to determine whether the increase of the sprouting ability in the case without the SMVN can compensate its absence regarding tumour growth.

\section{Cell resistance to hypoxia}

Tumour cells have the ability to survive under low oxygen concentrations. When the level of oxygen reaches the hypoxic threshold $\alpha_{p}$, proliferative cells switch to a quiescent state characterized by a reduced metabolism with reduced oxygen consumption. If the oxygen level further decreases, below the threshold $\alpha_{q}$, quiescent cells die of severe hypoxia. However these thresholds vary depending on the tumour cell phenotype. The most aggressive phenotypes are characterized by a greater resistance to hypoxia, where the cells can survive with extremely low level of oxygen.

For the two considered tissue cases, the threshold $\alpha_{q}$ between quiescence and necrosis taken for the simulations is different. In the weakly vascularised tissue (i.e. without SMVN), the tumour cells necessarily need to be more resistant for the tumour to start developing. Indeed proliferative tumour cells are initially implanted at some distance from a feeding vessel where the oxygen concentration is very low. Consequently, if the threshold $\alpha_{q}$ is too high, the proliferative cells directly die through necrosis. This requires to lower $\alpha_{q}$ below the concentration level to which the implanted proliferative cells are initially exposed to. Since it is very low (below 0.025), $\alpha_{q}$ has been fixed to 0. This means that the tumour cells are highly resistant and they can only die when there is no oxygen at all (although they also die through apoptosis when space is lacking for cell division). In the other tissue case, where the SMVN is present, the oxygen level in the tissue is much higher and the value of $\alpha_{q}$ can be elevated. It was then fixed to $\alpha_{p} / 10$. Tumour cells are less resistant in that case.

In the following simulation results, variations in the proliferative threshold $\alpha_{p}$ were considered. The 
different thresholds examined are presented in figure 12 using the same colour code for oxygen as in the earlier simulations. Figures 12a-b show the evolution over time of the quantity of living tumour cells (proliferative and quiescent) for the two tissue cases (without and with SMVN). The ratios for each cell state, proliferative, quiescent and necrotic are plotted separately in Fig 12c-d, e-f and g-h respectively.

Only when $\alpha_{p}$ is very high (above 2.5), meaning that the tumour cells need a lot of oxygen to remain proliferative, the population of living cells can be totally eradicated (Fig. 12a-b). In both tissue case, the proliferative cells implanted immediately become quiescent (Fig. 12c-d). They can only survive in this state for a limited time of 10 days before dying through necrosis (Fig. 12e-h). In ten days, the vascular network recruited could not cover the oxygen needs of the quiescent cell population to bring it back to proliferation and the cells eventually die. We note that this oxygen threshold was deliberately chosen to be above the oxygen carrying capacity of the vascular network to illustrate this extreme situation.

At the other extreme, when $\alpha_{p}$ is very low $\left(\alpha_{p}=0.0025\right)$, which corresponds to very resistant cells able to proliferate under low oxygen conditions, no necrosis is observed (Fig. 12g-h). In the presence of the SMVN, the cells do not even become quiescent (Fig. 12f), they remain proliferative and can only die through apoptosis when space is lacking for cell division (Fig. 12d).

For an intermediate proliferative threshold $\left(\alpha_{p}=0.25\right)$, which corresponds to an oxygen level close to the delivery amount from the neovessels, transitions between the three different cell states occur in both tissue case (with or without SMVN).

\section{Sprouting ability of the vascular network}

Endothelial cells forming the vessel walls detach and migrate to form some sprouts under the effect of VEGF. We therefore assumed in this model that vessels sprouting occurs with a probability which depends on the VEGF concentration. The reference probability of sprouting ranges from 0.0025 to 0.025 per day for VEGF concentrations ranging from 0.005 to 0.5 nu.day ${ }^{-1}$ (see Table c). We study here the impact of changes in these probabilities by increasing or decreasing them by a factor 10 .

The increase probability of vessels sprouting is expected to induce faster vascular growth and higher vessels density. In order to measure these two effects an observation window, adjacent to the tumour (at time $t=30$ days), is defined (Fig. 8). The total cumulated length of vessels measured in this window, for different times, gives the evolution of the vessels density. Displacement of the observation window, 
further away from the tumour, allows one to evaluate the time it takes for the new vessels to develop in this zone. We can thus estimate the propagation rate of angiogenesis.

Figure 13 compares the tissue cases without and with SMVN. When the SMVN is present, angiogenesis is delayed. Whereas the first sprouts appear in a few days without SMVN, it takes about a week for the SMVN to start sprouting. The existing vasculature slows down the occurrence of hypoxia, that is the signal which triggers vascular growth.

For the highest sprouting probability $(\mathrm{f}=10$ ), the vessel density (observed in zone 1 ) saturates in about 10 days after angiogenesis started from the SMVN, whereas it takes two times longer to reach saturation without the SMVN. Saturation has not yet occurred in zone 2 at time $t=30$ days for the two tissue cases, even for the highest sprouting probability. The propagation rate of angiogenesis, that we expect quicker without SMVN, is not significantly different between the two tissue cases. Angiogenesis even starts developing later when there is no SMVN, later than 10 days for the smallest sprouting probability $(\mathrm{f}=0.1$ and $\mathrm{f}=1)$, compared to the case with SMVN. Only when the sprouting probability is very high $(\mathrm{f}=10)$, angiogenesis occurs faster when there is no SMVN.

The sprouting probability is "expressed" only when there is VEGF, that is when tumour cells are hypoxic. It therefore takes more time to reach hypoxia when the SMVN is present. However once hypoxia starts, the SMVN which has a higher length of vessels from which sprouts can form, can grow quickly. To sum up, the hypoxic signal is long to occur but the response of the tissue is fast thanks to the existing SMVN. In the other tissue case, where there is no SMVN, the hypoxic signal is triggered almost immediately, but the response of the tissue is slow due to the time it takes to grow new vessels from the few existing ones.

The consequence of this effect on the evolution of the living tumour cell quantities is visible in figure 14 . When the SMVN is present (Fig. 14b), the sprouting probability of the vascular network has no influence on the evolution of the population of tumour cells, since this network is able to adapt instantaneously. In the other hand, when there is no SMVN, the sprouting probability of the vascular network affects the evolution of the tumour cell quantity, where an increase probability of sprouting leads to a faster growth of the tumour cells (Fig. 14a). 


\section{Discussion}

The ultimate aim of this work is to produce a computational model to mimic the development of a tumour mass introduced in a dorsal skinfold chamber so as to be able to virtually test a range of therapies. A first step was to assemble the bricks of the model and to define the most pertinent model hypotheses that take into account and match the data that can be obtained from the experimental setup. The question of the SMVN arose from reading the literature of the existing models and from our preliminary experimental observations that could not provide a clear answer. The aim of this contribution was therefore to investigate the necessity of taking this SMVN into account in the computational model, either explicitly, implicitly (indirectly) or not at all.

We defined the SMVN as the regular pre-existing and healthy microvascular network that ensures physiological oxygenation of the tissue. We characterized this network as a regular grid of capillaries having a diameter of about $8 \mu \mathrm{m}$ and with an interspace distance of $100 \mu \mathrm{m}$ that we based on our experimental measurements. Those are in agreement with values reported in the literature (Secomb et al., 2004; Welter et al., 2008, 2009). Based on a purely qualitative basis, simulations of angiogenesis realised without taking into account the SMVN are in good agreement with the experimental observations. The simulated vascular networks resemble those obtained in these experiments. However, since the SMVN is not visible at the macroscopic scale, that is at the scale of the dorsal window, it remains difficult to assess its role. To that end, simulations have been realised by systematically comparing two cases, with or without the SMVN that we superimposed to the main vasculature extracted from an experimental case. The aim is then to identify the main differences between the two cases if any. These two cases correspond to a homogeneously or a weakly vascularised tissue respectively.

Our results show that taking into account the SMVN leads to a well structured tumour with a large necrotic core, covered by a ring of quiescent cells and surrounded by a rim of proliferative cells. This hierarchical structure leads to efficient tumour growth, since quiescent cells maintain a stable VEGF signal that allows them to recruit continuously more vessels which ensure the sustained proliferation of the rim cells. In the other case, when the SMVN is not present, a different scenario is observed. The lack of oxygenation in the tissue at the initial stage induces a delayed development of the tumour. Then, the heterogeneous oxygen distribution in the tissue leads to a more anarchic development of the tumour mass due to outbursts of VEGF signals. The tumour is then mainly characterized by the presence of several isolated necrotic clusters of smaller size than in the previous tissue case where the unique necrotic core is big. Whereas the necrotic state is dominating the tumour cell population (after the first month) 
when the SMVN is present, the proliferative state dominates when the SMVN is absent. Although the tumour grows slowly in this case, it is however more active since most of its mass is alive. Experimental verification is now required to determine which scenario is most valid. For example, immunostaining of the tumour mass to map the distribution of the tumour cell types would be one mean to do this.

In a second part, parameters that are known to strongly influence the tumour evolution have been tested in the two tissue cases (i.e. with or without SMVN). The first parameter concerns the cell capacity to resist to hypoxia. This parameter relates to the definition of the cell phenotype and allows us to consider a broader spectrum of tumour growth behaviours that can be encountered. We observed that for a given phenotype, the tumour evolution remains mainly directed by the intrinsic properties of the cell population rather than on the initial degree of vascularisation of the tumour environment. In other words, higher resistance to hypoxia favours tumour growth whatever the tissue case. We then tested the parameter that regulates the sprouting ability of the vascular network, with the back idea that increasing this probability in the case without SMVN would compensate its absence. This was indeed the case concerning the speed of tumour growth that could be increased. In the other hand, alterations of the sprouting capacity has no influence when the SMVN is present due to saturation of the vessels density.

Since well vascularised tissue such as skin tissue are oxygen consuming, the role of the SMVN is essential in their physiological oxygenation. Indeed, in consequence of the relative low solubility of oxygen in tissue, vessels can only diffuse oxygen in a close neighbourhood around them (20 to $100 \mu \mathrm{m})$ if the cell consumption in oxygen is high (Secomb et al., 2004). Consequently, the presence of the SMVN in the model at least implicitly is required unless the tumour is already vascularised at the initial state of the model (Cai et al., 2011; Pons-Salort et al.). However, is it necessary to explicitly take it into account in computer simulations in order to realistically reflect the tumour evolution as we observe it in the dorsal skinfold chamber? We could consider not to represent the grid of vessels but to replace it by a physiologic ground level of oxygen throughout the tissue. This solution is not entirely satisfying since it cannot account for the changing conditions in the oxygen field. With a ground level of oxygen, hypoxia cannot be reached and the tumour cannot produce the VEGF that triggers angiogenesis. Consequently, local variations of the oxygen ground level have to be considered through oxygen consumption by at least the tumour cells. But in that case, oxygen needs to be regenerated to avoid complete tissue necrosis. Finding the right oxygen balance without the physical presence of the smallest vessel is therefore a complex issue.

Throughout the recent years, various models have been proposed that take more or less explicitly 
into account the SMVN. Zheng et al. (2005) presented a model where an oxygen basis is provided to the tissue by pre-existing capillaries. Additionally the extra-tumoral tissue is considered as non-oxygen consuming. This model has the benefit to reproduce an oxygen gradient from the tumour periphery to the core compatible with experimental observation. In that case, the SMVN and its evolution with time is non-explicitly considered and the model does not reflect the physical reality of oxygen consumption in healthy tissue. In the paper by Macklin et al. (2009) the SMVN is also considered implicitly in addition to the neovasculature issued from a parent vessel in the model. The degradation of the SMVN is assumed to be related to the presence of matrix degrading enzymes secreted by living tumour cells. This model integrates oxygen consumption of the healthy tissue. It is based on the assumption that neovessels issued from main vessels are stable contrary to the submicrovascular vessels which collapse in the tumour. The major limitation of the implicit integration of the SMVN is that the contribution of these pre-existing capillaries in the angiogenic process is not considered.

There are fewer theoretical models of tumour growth that explicitly integrate the SMVN. Among those models we have been especially interested by the models proposed by Bartha and Rieger (2006); Lee et al. (2006); Paul (2009); Welter et al. (2008). These models integrate the SMVN as a regular square grid formed by vessels having the same radius. Similarly, Welter et al. (2008) use a regular hexagonal pattern. Most models agree with a capillary interdistance of $100 \mu \mathrm{m}$ and we also took this value. The explicit integration of the SMVN gives access to a better description of the tumour environment to reflect the heterogeneous tumour development. Indeed, capillaries degradation is not homogeneous but depends on their stability. Capillaries with higher wall shear stress are not degraded in the tumour, this breaks the symmetry of tumour growth has we could see it in our simulations. More recent works of Welter et al. introduce varying vessels radii. These variations depend on the branching pattern and lead to a more selective collapse of tumour vessels (Welter et al., 2009; Welter and Rieger, 2010). Angiogenesis of the SMVN can also be considered. Recent works integrate this aspect, though in a simplified way since sprouting vessels direction is not influenced by VEGF gradients (Bartha and Rieger, 2006; Welter et al., 2008, 2009; Welter and Rieger, 2010).

\section{Conclusion}

Taking into account the SMVN is obviously necessary to ensure physiological oxygenation of the tissue. Its explicit integration in theoretical models of tumour growth is relatively recent. These models have highlighted its role. In this paper our aim was to determine whether it was necessary to have such an explicit integration of the SMVN to reproduce tumour growth as we observed it in a dorsal skinfold chamber on the mouse. We reach the conclusion that the physical presence of the smallest vessels is 
required to account for the rapid response of the vasculature to tumour hypoxia and to inversely reflect hypoxia induced by collapsing vessels. However the integration as a regular grid is not necessarily the best choice. We could incorporate instead a more detailed vascular network, rather than exclusively retaining the main visible vessels at the dorsal window scale such as we did. Moreover, one important aspect that we have not considered is vessel remodelling. Although we considered flow selection on the capillaries, we have not integrated the effects of flow variations induced by vessels collapsing. These effects lead to vessels dilation or contraction and reinforce the role of vessels selection. We intend to especially focus on this aspect for an improved version of a computational model of tumour growth. This will be our next step towards our goal of providing a virtual tumour, in the dorsal skinfold chamber context, as a tool to investigate therapy.

\section{Acknowledgements}

This work was funded by CNRS (PEPS-INS2I 2010-2011) and by the French National Network and the Rhône-Alpes Institute for Complex Systems (RNCS and IXXI respectively).

\section{References}

Alarcòn, T., Byrne, H.M., Maini, P.K., 2003. A cellular automaton model for tumour growth in inhomogeneous environment. J Theor Biol 225, 257-274.

Anderson, A.R., Chaplain, M.A., 1998. Continuous and discrete mathematical models of tumor-induced angiogenesis. Bull Math Biol 60, 857-899.

Asaishi, K., Endrich, B., Gtz, A., Messmer, K., 1981. Quantitative analysis of microvascular structure and function in the amelanotic melanoma a-mel-3. Cancer Res 41, 1898-1904.

Balding, D., McElwain, D.L., 1985. A mathematical model of tumour-induced capillary growth. J Theor Biol 114, 53-73.

Bartha, K., Rieger, H., 2006. Vascular network remodeling via vessel cooption, regression and growth in tumors. J Theor Biol 241, 903-918.

Bauer, A.L., Jackson, T.L., Jiang, Y., 2007. A cell-based model exhibiting branching and anastomosis during tumor-induced angiogenesis. Biophys J 92, 3105-3121.

Burton, A.C., 1966. Rate of growth of solid tumours as a problem of diffusion. Growth 30, 157-176. 
Byrne, H.M., 2010. Dissecting cancer through mathematics: from the cell to the animal model. Nat Rev Cancer 10, 221-230.

Cai, Y., Xu, S., Wu, J., Long, Q., 2011. Coupled modelling of tumour angiogenesis, tumour growth and blood perfusion. J Theor Biol In Press, Uncorrected Proof, --

Chaplain, M.A.J., McDougall, S.R., Anderson, A.R.A., 2006. Mathematical modeling of tumor-induced angiogenesis. Annu Rev Biomed Eng 8, 233-257.

Dedeugd, C., Wankhede, M., Sorg, B.S., 2009. Multimodal optical imaging of microvessel network convective oxygen transport dynamics. Appl Opt 48, D187-D197.

Dellian, M., Witwer, B.P., Salehi, H.A., Yuan, F., Jain, R.K., 1996. Quantitation and physiological characterization of angiogenic vessels in mice: effect of basic fibroblast growth factor, vascular endothelial growth factor/vascular permeability factor, and host microenvironment. Am J Pathol 149, 59-71.

Frieboes, H.B., Edgerton, M.E., Fruehauf, J.P., Rose, F.R.A.J., Worrall, L.K., Gatenby, R.A., Ferrari, M., Cristini, V., 2009. Prediction of drug response in breast cancer using integrative experimental/computational modeling. Cancer Res 69, 4484-4492.

Fukumara, D., Jain, R.K., 2008. Imaging angiogenesis and the microenvironment. APMIS 116, 695-715.

Gatenby, R.A., Silva, A.S., Gillies, R.J., Frieden, B.R., 2009. Adaptive therapy. Cancer Res 69, 4894-4903.

Goldman, D., Popel, A.S., 2000. A computational study of the effect of capillary network anastomoses and tortuosity on oxygen transport. J Theor Biol 206, 181 - 194.

Greenspan, H.P., 1972. Models for the growth of a solid tumour by diffusion. Stud Appl Math L1, $317-340$.

Koehl, G.E., Gaumann, A., Geissler, E.K., 2009. Intravital microscopy of tumor angiogenesis and regression in the dorsal skin fold chamber: mechanistic insights and preclinical testing of therapeutic strategies. Clin Exp Metastasis 26, 329-344.

Lee, D.S., Rieger, H., Bartha, K., 2006. Flow correlated percolation during vascular remodeling in growing tumors. Phys Rev Lett 96, 058104.

Lehr, H.A., Leunig, M., Menger, M.D., Nolte, D., Messmer, K., 1993. Dorsal skinfold chamber technique for intravital microscopy in nude mice. Am J Pathol 143, 1055-1062. 
Macklin, P., McDougall, S., Anderson, A., Chaplain, M., Cristini, V., Lowengrub, J., 2009. Multiscale modelling and nonlinear simulation of vascular tumour growth. J Math Biol 58, 765-798.

Maurin, M., Stephan, O., Vial, J.-C., van der Sanden, B., 2011. Deep in vivo two-photon imaging of blood vessels with a new dye encapsulated in pluronic nanomicelles.. J Biomed Opt 16, 036001.

McDougall, S.R., Anderson, A.R.A., Chaplain, M.A.J., 2006. Mathematical modelling of dynamic adaptive tumour-induced angiogenesis: clinical implications and therapeutic targeting strategies. J Theor Biol 241, 564-589.

McDougall, S.R., Chaplain, M.A.J., ephanou, A.S., Anderson, A.R.A., 2010. Modelling the impact of pericyte migration and coverage of vessels on the efficacy of vascular disrupting agents. Math Model Nat Phenom 3, 1-41.

Owen, M.R., Alarcòn, T., Maini, P.K., Byrne, H.M., 2009. Angiogenesis and vascular remodelling in normal and cancerous tissues. J Math Biol 58, 689-721.

Paul, R., 2009. Flow-correlated dilution of a regular network leads to a percolating network during tumor-induced angiogenesis. Eur Phys J E Soft Matter 30, 101-114. 10.1140/epje/i2009-10513-8.

Perfahl, H., Byrne, H.M., Chen, T., Estrella, V., Alarcn, T., Lapin, A., Gatenby, R.A., Gillies, R.J., Lloyd, M.C., Maini, P.K., Reuss, M., Owen, M.R., 2011. Multiscale modelling of vascular tumour growth in 3d: the roles of domain size and boundary conditions. PLoS One 6, e14790.

Plank, M.J., Sleeman, B.D., Jones, P.F., 2004. A mathematical model of tumour angiogenesis, regulated by vascular endothelial growth factor and the angiopoietins. J Theor Biol 229, 435-454.

Pons-Salort, M., van der Sanden, B., Stéphanou, A., A computational framework to assess the efficacy of cytotoxic molecules and vascular disrupting agents against solid tumours. Submitted 2011 .

Pries, A.R., Secomb, T.W., Gessner, T., Sperandio, M.B., Gross, J.F., Gaehtgens, P., 1994. Resistance to blood flow in microvessels in vivo. Circ Res 75, 904-915.

Qutub, A.A., Popel, A.S., 2009. Elongation, proliferation \& migration differentiate endothelial cell phenotypes and determine capillary sprouting. BMC Syst Biol 3, (13).

Secomb, T.W., Hsu, R., Park, E.Y.H., Dewhirst, M.W., 2004. Green's function methods for analysis of oxygen delivery to tissue by microvascular networks. Ann Biomed Eng 32, 1519-1529.

Shirinifard, A., Gens, J.S., Zaitlen, B.L., Poplawski, N.J., Swat, M., Glazier, J.A., 2009. 3d multi-cell simulation of tumor growth and angiogenesis. PLoS ONE 4, e7190. 
Stéphanou, A., McDougall, S.R., Anderson, A.R.A., Chaplain, M.A.J., 2005. Mathematical modelling of flow in 2d and 3d vascular networks: Applications to anti-angiogenic and chemotherapeutic drug strategies. Math Comput Model 41, 1137-1156.

Stéphanou, A., McDougall, S.R., Anderson, A.R.A., Chaplain, M.A.J., 2006. Mathematical modelling of the influence of blood rheological properties upon adaptative tumour-induced angiogenesis. Math Comput Model 44, 96 - 123.

Stokes, C.L., Lauffenburger, D.A., 1991. Analysis of the roles of microvessel endothelial cell random motility and chemotaxis in angiogenesis. J Theor Biol 152, 377-403.

Strieth, S., Eichhorn, M.E., Sauer, B., Schulze, B., Teifel, M., Michaelis, U., Dellian, M., 2004. Neovascular targeting chemotherapy: encapsulation of paclitaxel in cationic liposomes impairs functional tumor microvasculature. Int J Cancer 110, 117-124.

Strieth, S., Eichhorn, M.E., Werner, A., Sauer, B., Teifel, M., Michaelis, U., Berghaus, A., Dellian, M., 2008. Paclitaxel encapsulated in cationic liposomes increases tumor microvessel leakiness and improves therapeutic efficacy in combination with cisplatin. Clin Cancer Res 14, 4603-4611.

Tracqui, P., 2009. Biophysical models of tumour growth. Reports on Progress in Physics 72, 056701.

Welter, M., Bartha, K., Rieger, H., 2008. Emergent vascular nerwork inhomogenities and resulting blood flow patterns in a growing tumor. J Theor Biol 250, 257-280.

Welter, M., Bartha, K., Rieger, H., 2009. Vascular remodelling of an arterio-venous blood vessel network during solid tumour growth. J Theor Biol 259, 405-422.

Welter, M., Rieger, H., 2010. Physical determinants of vascular network remodeling during tumor growth. Eur Phys J E 33, 149-163.

Wu, F.T.H., Stefanini, M.O., Gabhann, F.M., Popel, A.S., 2009. A compartment model of vegf distribution in humans in the presence of soluble vegf receptor-1 acting as a ligand trap. PLoS One 4, (4).

Zheng, X., Wise, S.M., Cristini, V., 2005. Nonlinear simulation of tumor necrosis, neo-vascularization and tissue invasion via an adaptive finite-element/level-set method. Bull Math Biol 67, 211-259. 


\section{Tables}

Table 1

Vascular network and blood pressure

\begin{tabular}{|c|c|c|c|c|}
\hline Parameter & Unit & Value & Significance & Source \\
\hline$t_{E C}^{(\text {switch })}$ & $(h)$ & 24 & Time limit for vessel tumour sprouting & (Welter et al., 2009) \\
\hline$t_{E C}^{(\text {death })}$ & $(h)$ & 60 & Mean survival time of unstable segment & - \\
\hline$\tau^{(\mathrm{coll})}$ & $\mathrm{Pa}$ & 4 & Wall shear stress threshold for vessel's collapse & Estimated \\
\hline$P_{S_{1}}$ & $P a$ & 10000 & Pressure at the outer node of $S_{1}$ & Estimated \\
\hline$P_{S_{3}}$ & $P a$ & 10000 & Pressure at the outer node of $S_{3}$ & Estimated \\
\hline$P_{S_{7}}$ & $\mathrm{~Pa}$ & 100 & Pressure at the outer node of $S_{7}$ & Estimated \\
\hline$P_{S_{8}}$ & $\mathrm{~Pa}$ & 4000 & Pressure at the outer node of $S_{8}$ & Estimated \\
\hline$P_{S_{10}}$ & $\mathrm{~Pa}$ & 8500 & Pressure at the outer node of $S_{10}$ & Estimated \\
\hline$P_{S_{11}}$ & $\mathrm{~Pa}$ & 9000 & Pressure at the outer node of $S_{11}$ & Estimated \\
\hline \multicolumn{5}{|c|}{ Matrix fibre and matrix degrading enzyme } \\
\hline Parameter & Unit & Value & Significance & Source \\
\hline$D$ & $m m^{2} \cdot h^{-1}$ & $10.4 \times 10^{-3}$ & Enzyme diffusion coefficient & Estimated \\
\hline$\beta$ & $h^{-1}$ & 0 & Fibre production rate by endothelial cells & - \\
\hline$\gamma$ & $h^{-1}$ & $2.61 \times 10^{-4}$ & Fibre degradation rate by the enzymes & Estimated \\
\hline$\alpha$ & $h^{-1}$ & 130 & Enzyme production rate by an endothelial tip cell & Estimated \\
\hline$v$ & $h^{-1}$ & 1.30 & Enzyme spontaneous degradation rate & Estimated \\
\hline \multicolumn{5}{|l|}{ VEGF } \\
\hline Parameter & Unit & Value & Significance & Source \\
\hline$D_{V}$ & $m m^{2} \cdot h^{-1}$ & 0.104 & VEGF diffusion coefficient & (Stéphanou et al., 2005) \\
\hline$\lambda$ & $h^{-1}$ & 0.650 & Spontaneous degradation rate of VEGF & (Bauer et al., 2007) \\
\hline$\eta$ & $h^{-1}$ & 1 & Uptake rate of VEGF by endothelial cells & (Bauer et al., 2007) \\
\hline$V_{\max }$ & $h^{-1}$ & 0.06 & Maximum VEGF absorption by a portion of vessel & (Bauer et al., 2007) \\
\hline$V_{\text {emit }}$ & $n . u \cdot s^{-1}$ & 0.0145 & VEGF emission rate by a quiescent cell & - \\
\hline \multicolumn{5}{|l|}{ Oxygen } \\
\hline Parameter & Unit & Value & Significance & Source \\
\hline $\mathrm{D}_{\mathrm{O}_{2}}$ & $m m^{2} \cdot s^{-1}$ & $2.41 \times 10^{-3}$ & Oxygen diffusion coefficient & (Goldman and Popel, 2000) \\
\hline$\gamma_{p}$ & $s^{-1}$ & $3.11 \times 10^{-2}$ & Permeability to oxygen of the vessel walls & - \\
\hline$k_{P}$ & $s^{-1}$ & 2 & consumption rate of oxygen for proliferative tumour cells & Estimated \\
\hline$k_{N}$ & $s^{-1}$ & 1.57 & consumption rate of oxygen for normal cells & Estimated \\
\hline$k_{Q}$ & $s^{-1}$ & 1 & consumption rate of oxygen for quiescent tumour cells & Estimated \\
\hline \multicolumn{5}{|c|}{ Tumour cells } \\
\hline Parameter & Unit & Value & Significance & Source \\
\hline$\alpha_{p}$ & & 0.25 & Oxygen threshold for quiescence & - \\
\hline \multirow[t]{2}{*}{$\alpha_{q}$} & & 0 or 0.025 & Oxygen threshold for necrosis & \\
\hline & & & without or with SMVN & - \\
\hline$T_{\text {limit }}$ & days & 10 & Time after which a hypoxic cell dies by necrosis & Estimated \\
\hline$t_{\text {apop }}$ & $h$ & 5 & Time for macrophages cleaning of dead cells & Estimated \\
\hline$t_{G_{1}}$ & $h$ & 10 & $G_{1}$ phase duration & Estimated \\
\hline$t_{\text {cycle }}$ & $h$ & $22 \pm 2$ & Cell cycle duration & Estimated \\
\hline
\end{tabular}


Table 2

\begin{tabular}{cc}
\hline $\begin{array}{c}\text { VEGF quantity (normalized } \\
\text { unit) in a grid element }\end{array}$ & $\begin{array}{c}\text { Sprouting probability } \\
\text { per day }\end{array}$ \\
\hline ] $0.005-0.1]$ & 0.0025 \\
] $0.1-0.25]$ & 0.007 \\
] $0.25-0.5]$ & 0.015 \\
$>0.5$ & 0.025 \\
\hline
\end{tabular}




\section{Figures}

Figure 1

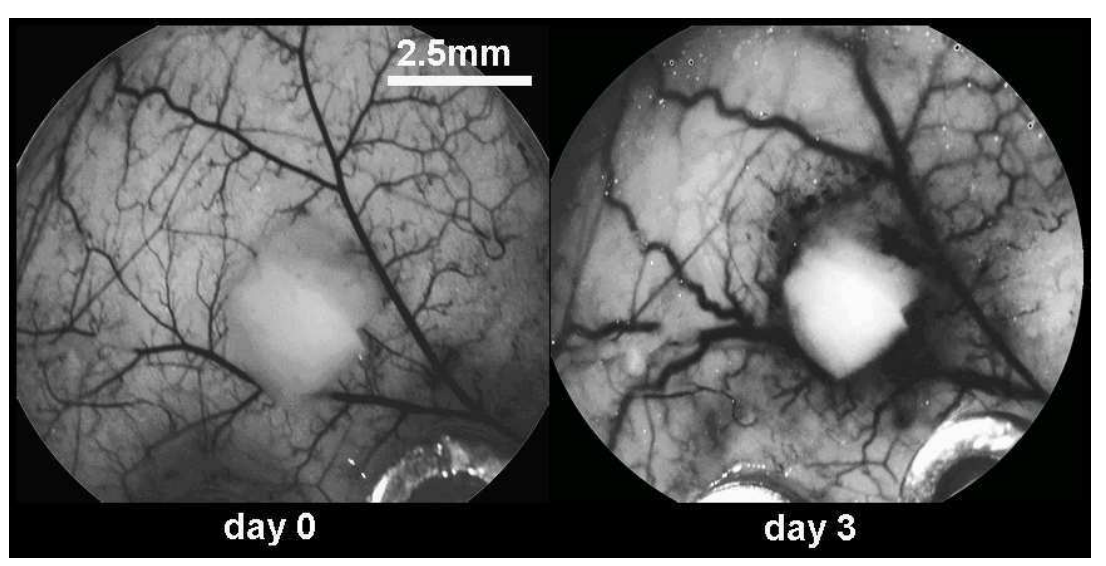

Figure 1: 
Figure 2
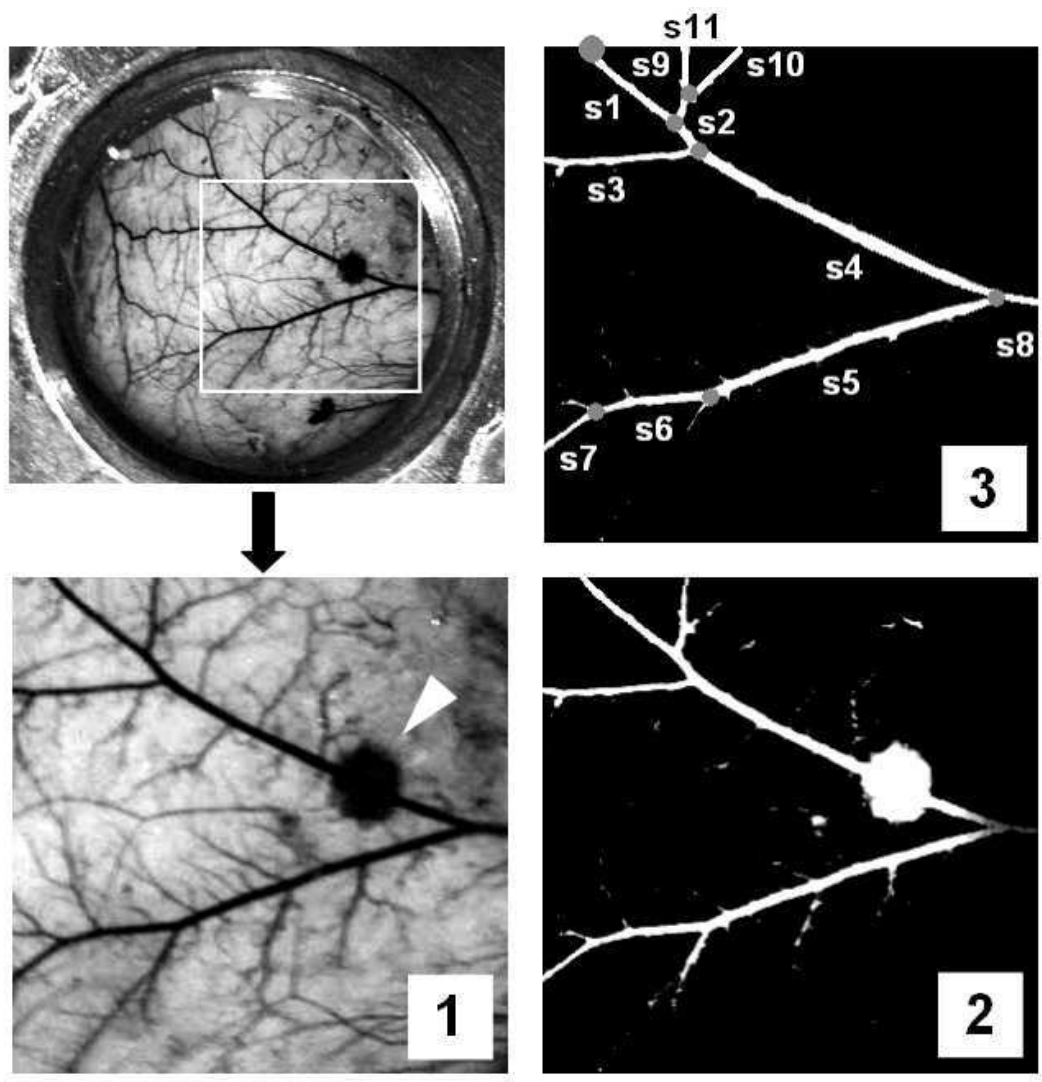

Figure 2: 
Figure 3

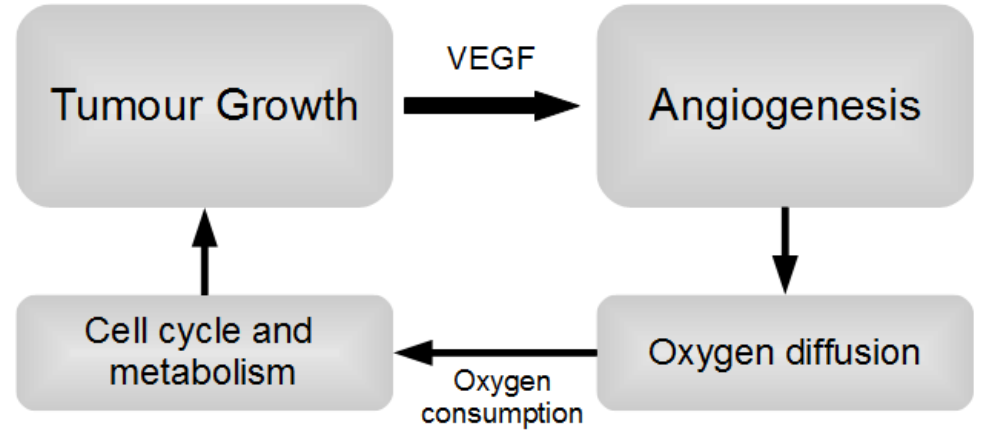

Figure 3: 
Figure 4

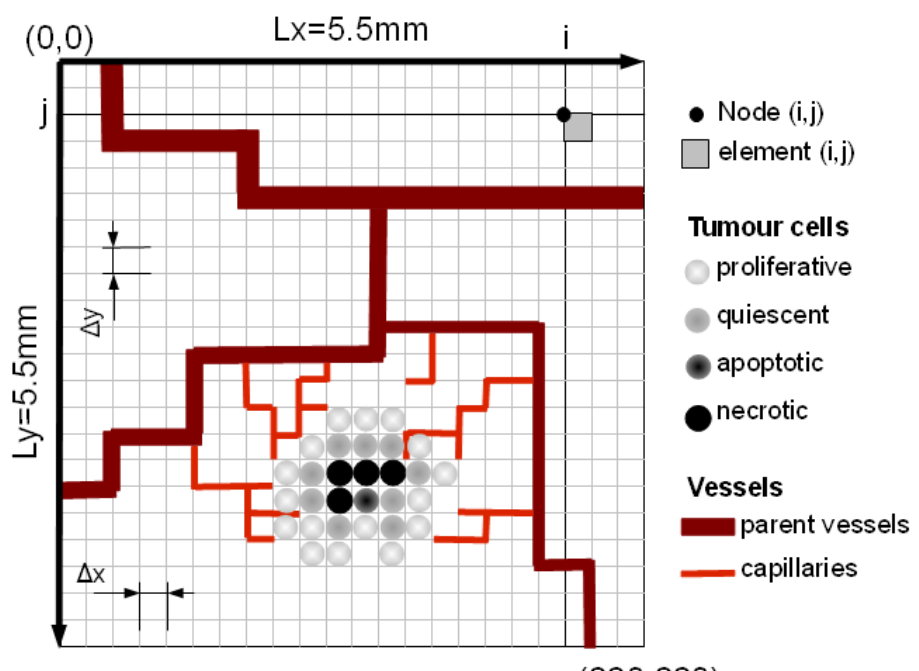

$(220,220)$

Figure 4: 
Figure 5

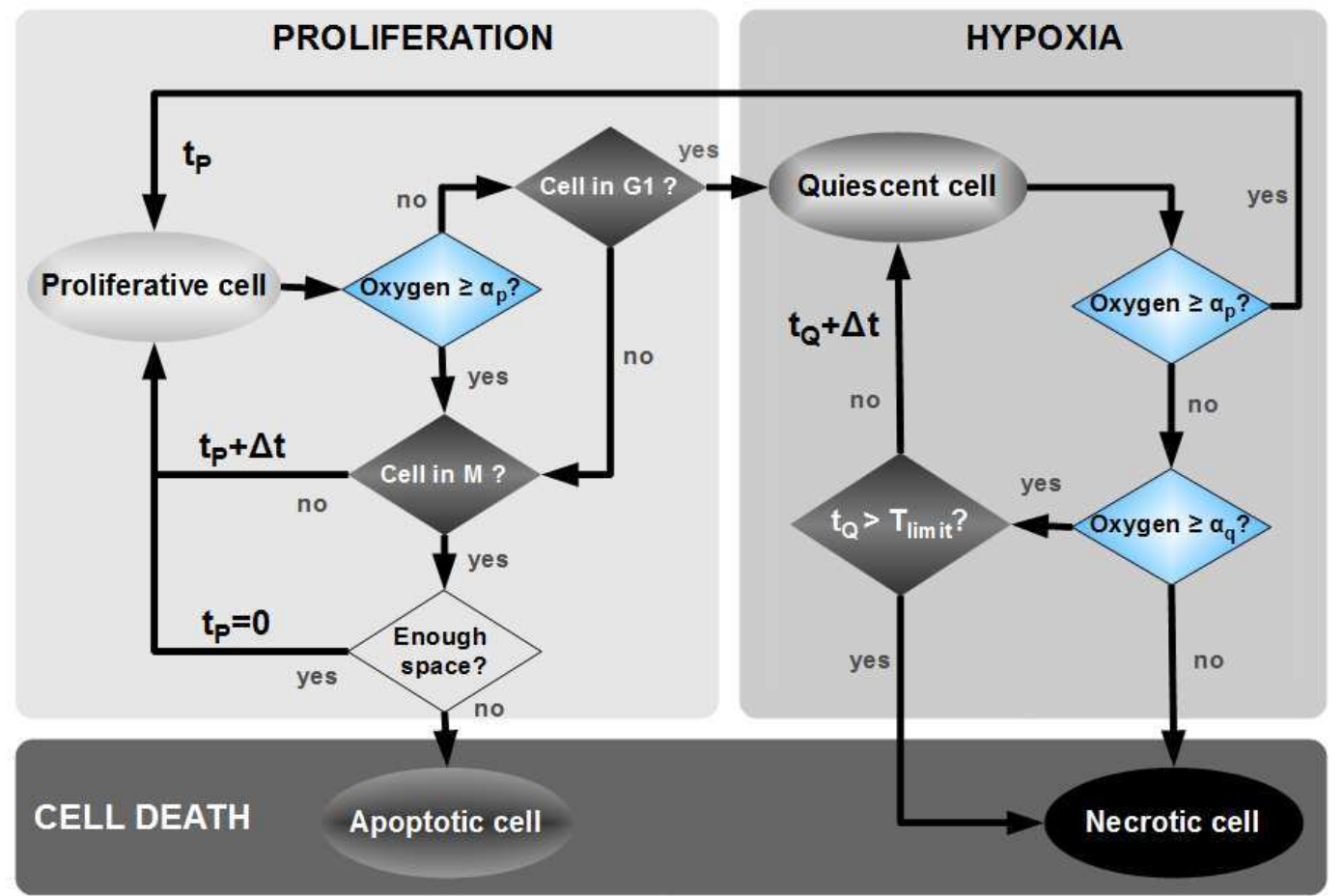

Figure 5: 
Figure 6
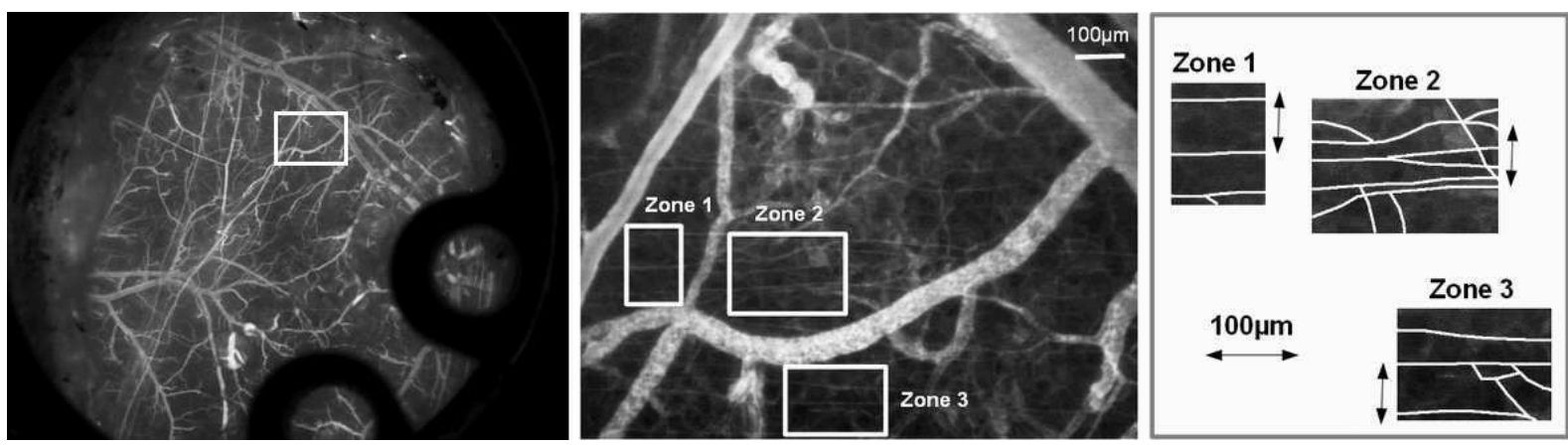

Figure 6: 
Figure 7
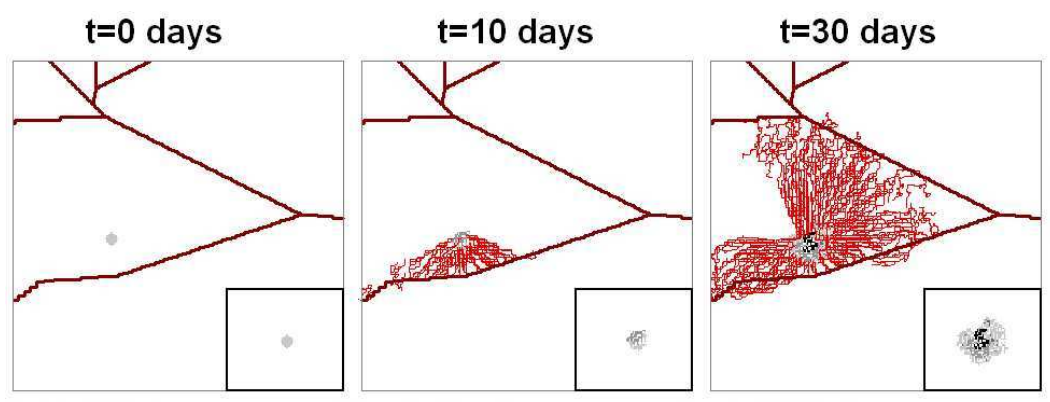

$\mathrm{t}=70$ days
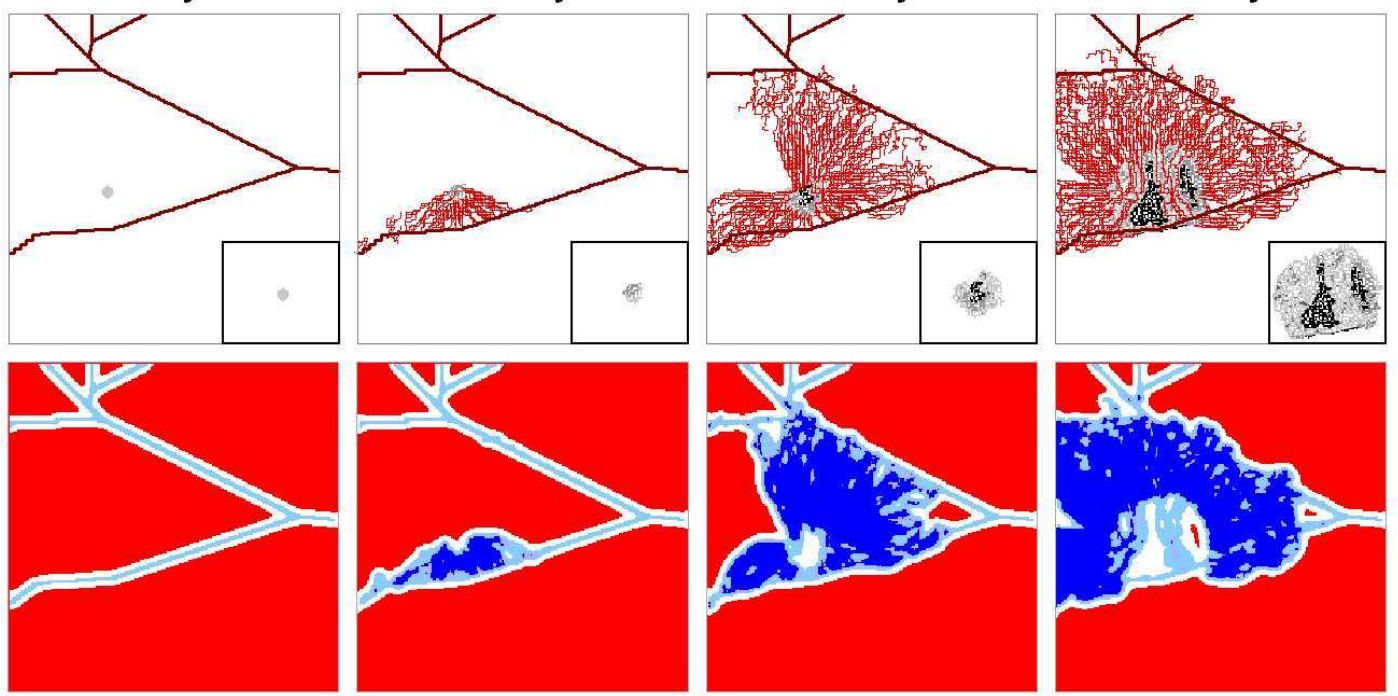

Tumour cells states

proliferativ

hypoxic

necrotic

Vessel radii

$<10 \mathrm{\mu m}$
$\geq 10 \mathrm{um}$
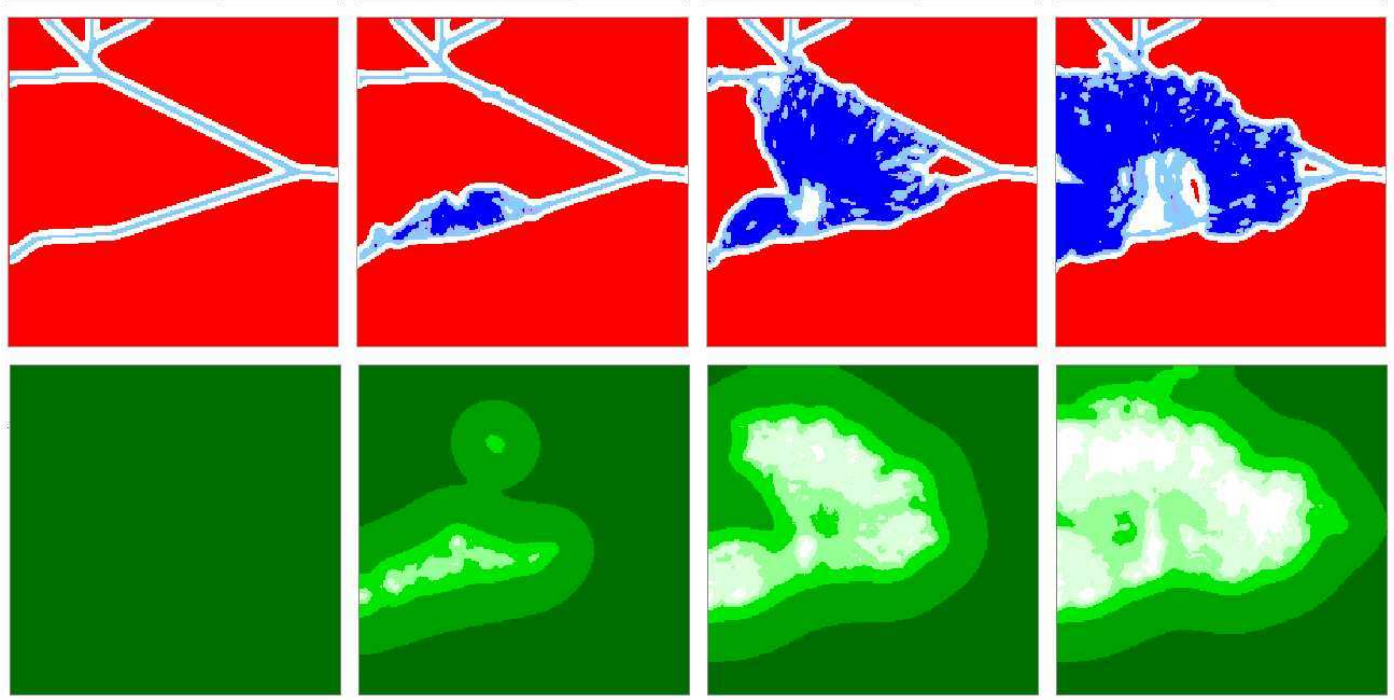

oxygen
concentration

in tissue

in tissue
(normalized)
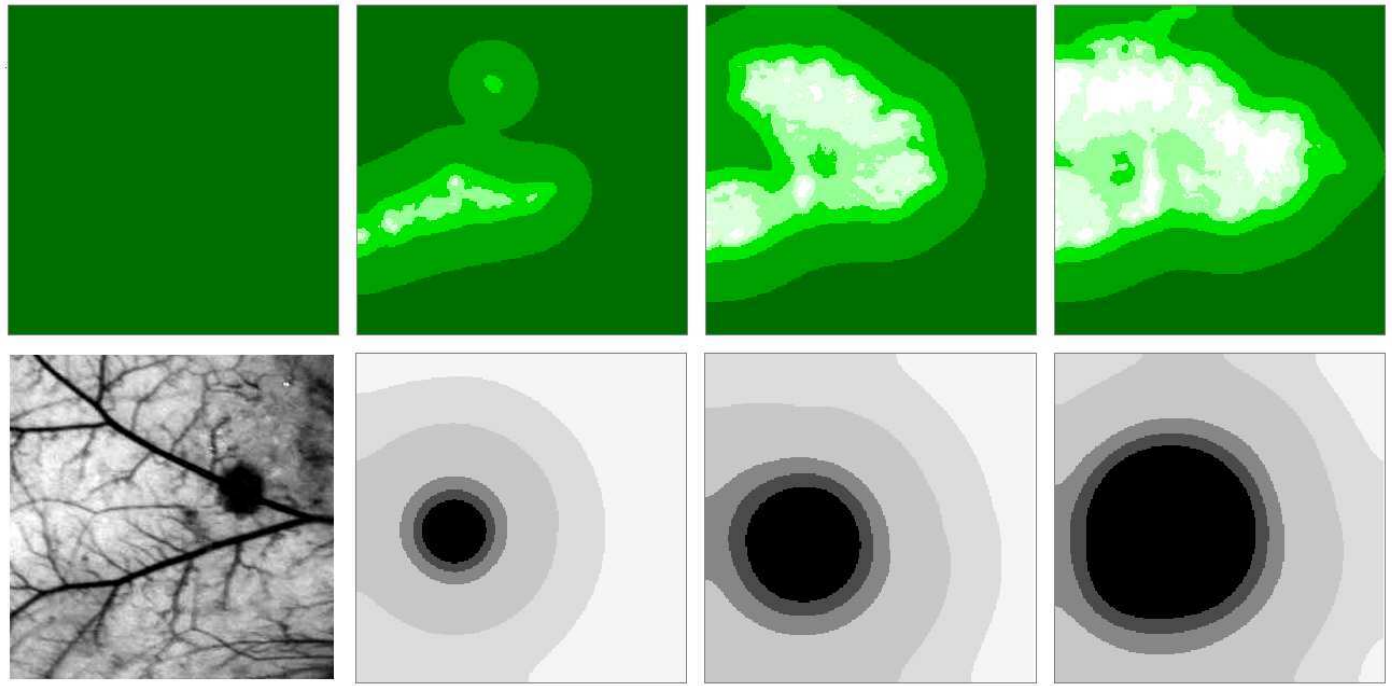

Degradation

of fibers

$<20 \%$

$<10 \%$
$<5 \%$
$<2.5 \%$

$<2.5 \%$
$0.2 \%$

Figure 7:

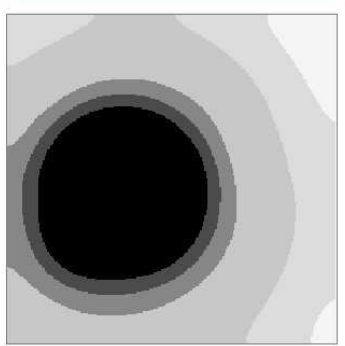

VEGF

concentration
(normalized)

0.3

0.5
-0.7


0.8 
Figure 8
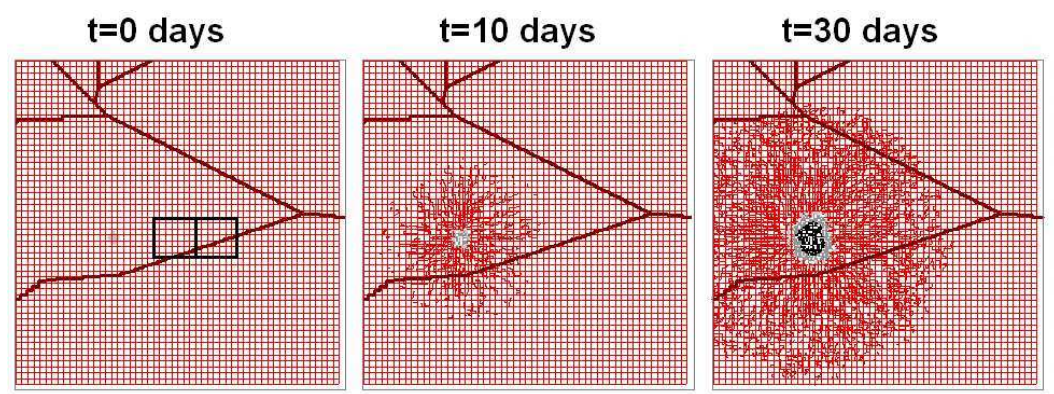

$\mathrm{t}=70$ days
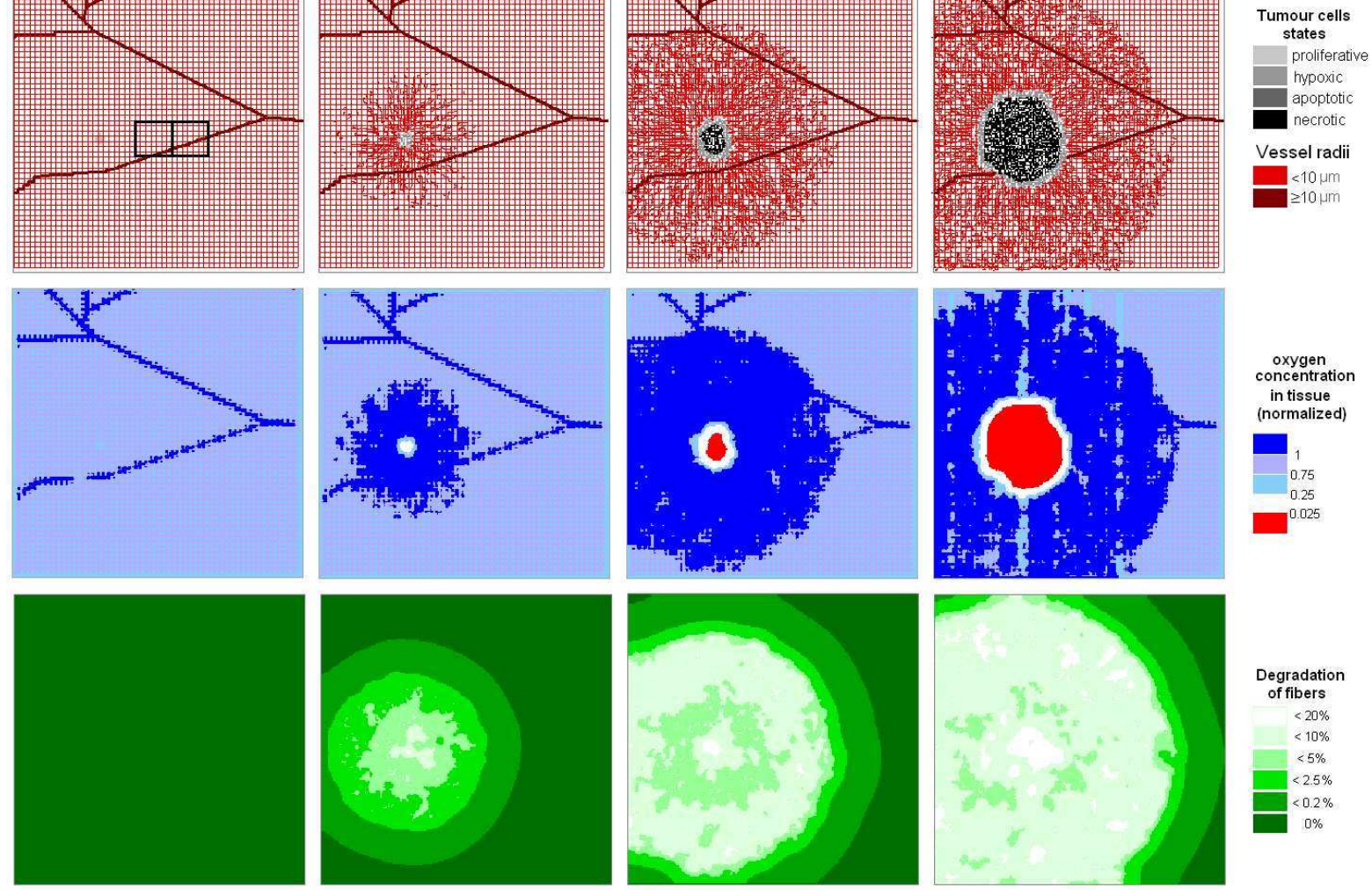

oxygen
concentration

in tissue
(normalized)
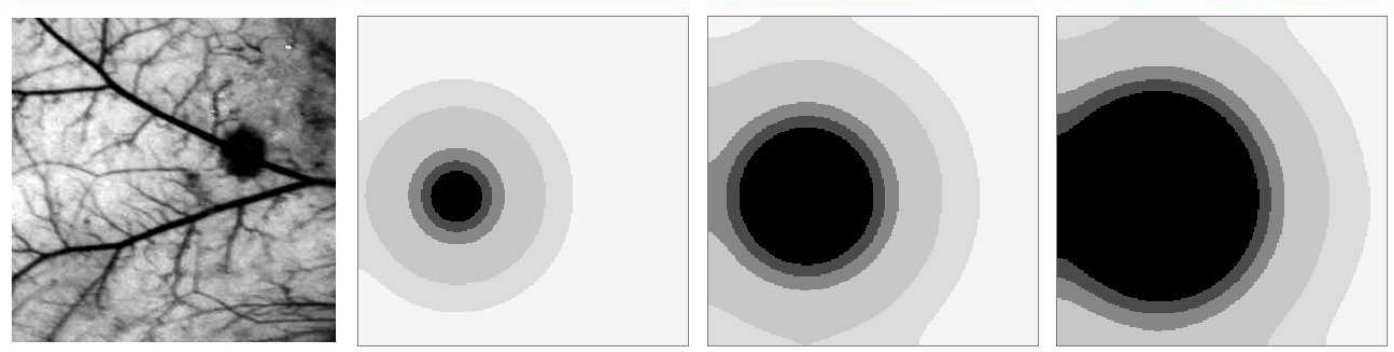

Degradation
of fibers

$<20 \%$

\begin{aligned}$< & <10 \% \\$\hline & $<5 \% \\ & <2.5 \%\end{aligned}$

\begin{tabular}{l}
$<2.5 \%$ \\
\hline $0.2 \%$ \\
\hline $0 \%$
\end{tabular}

Figure 8: 
Figure 9
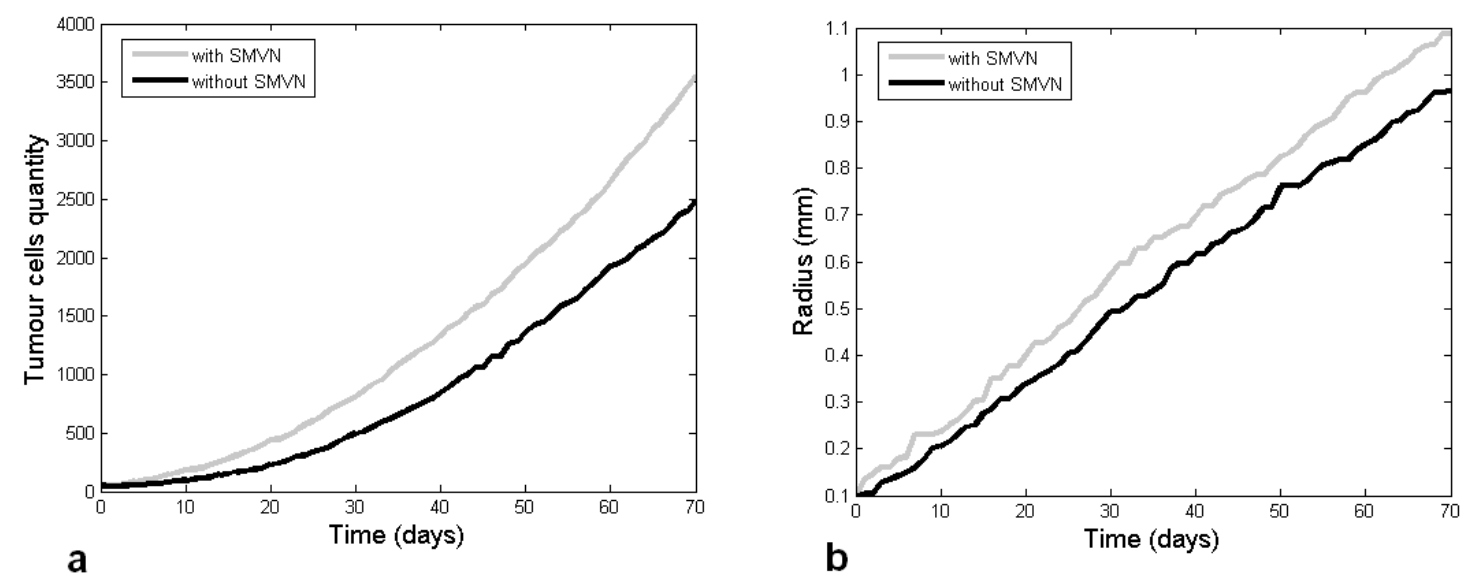

Figure 9: 
Figure 10
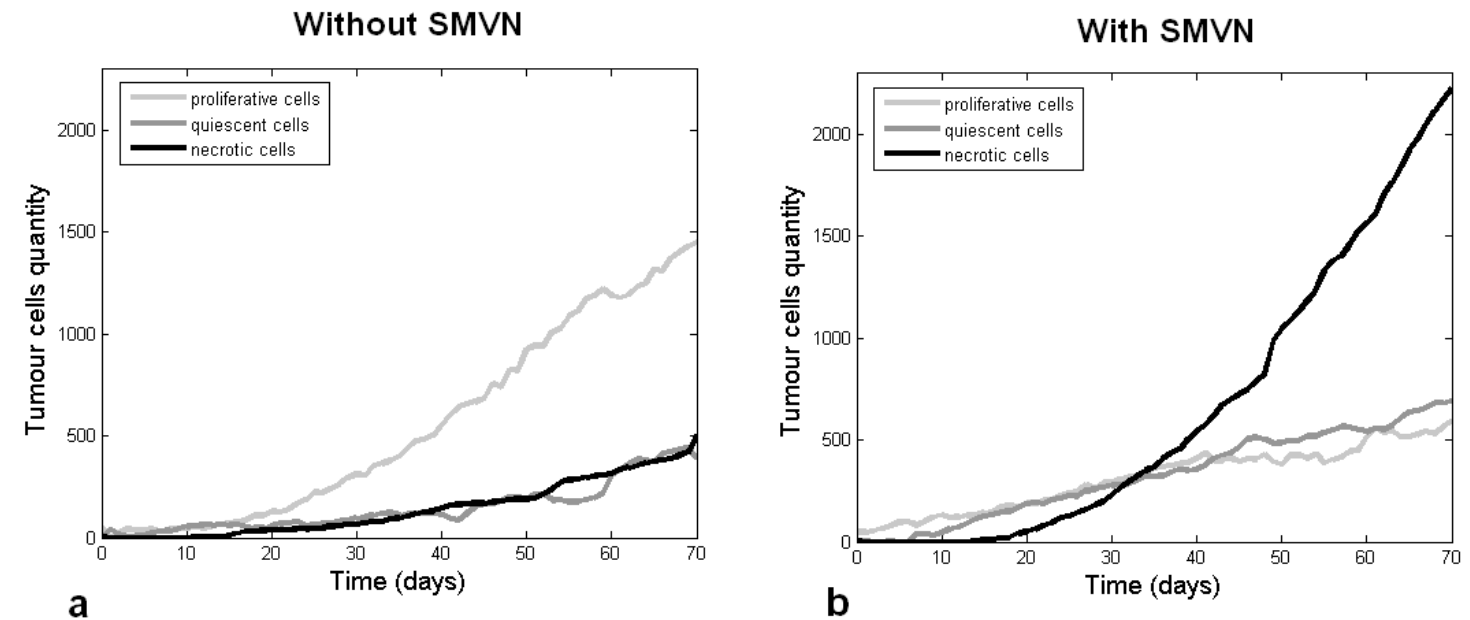

Figure 10: 
Figure 11
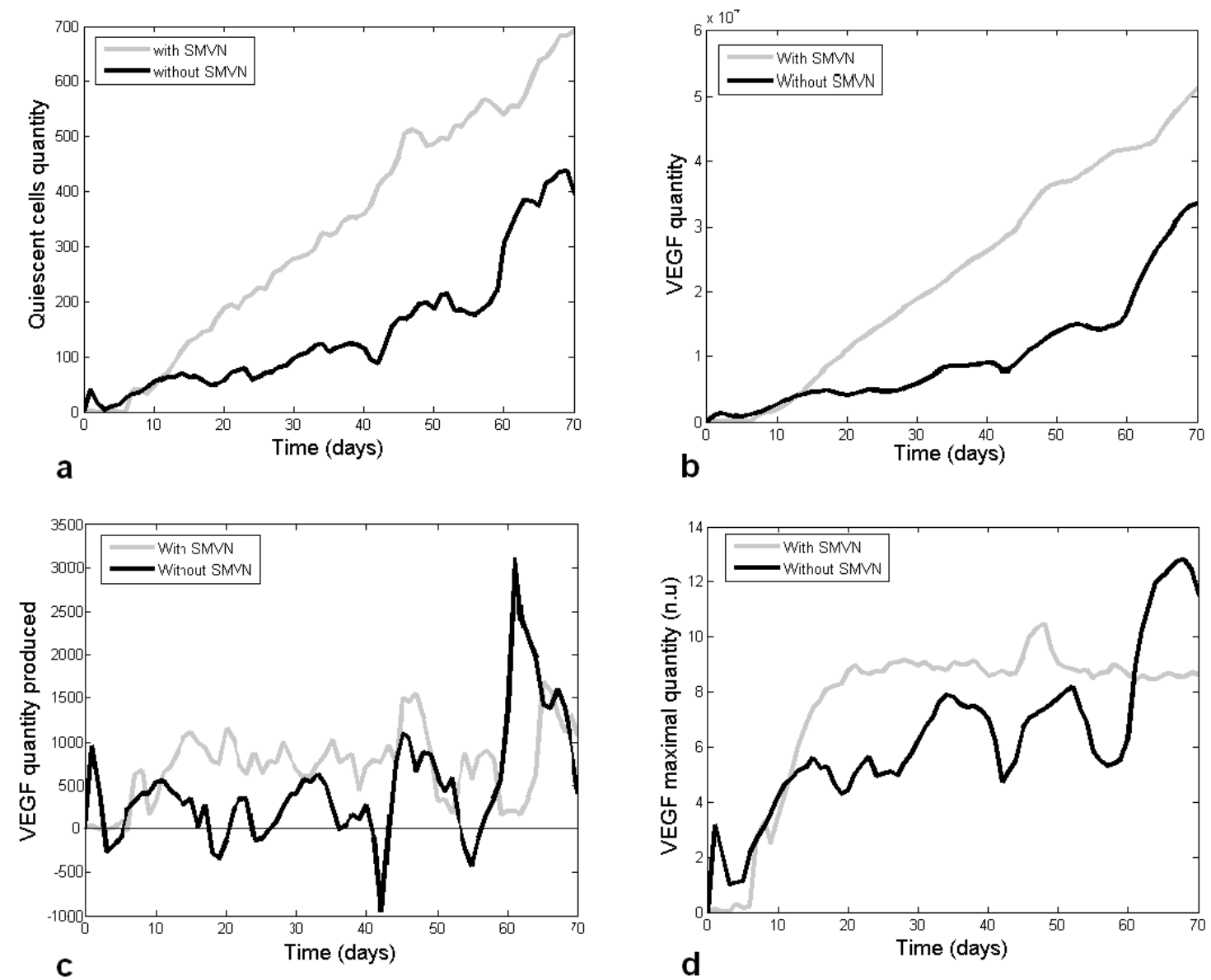

Figure 11: 
Figure 12
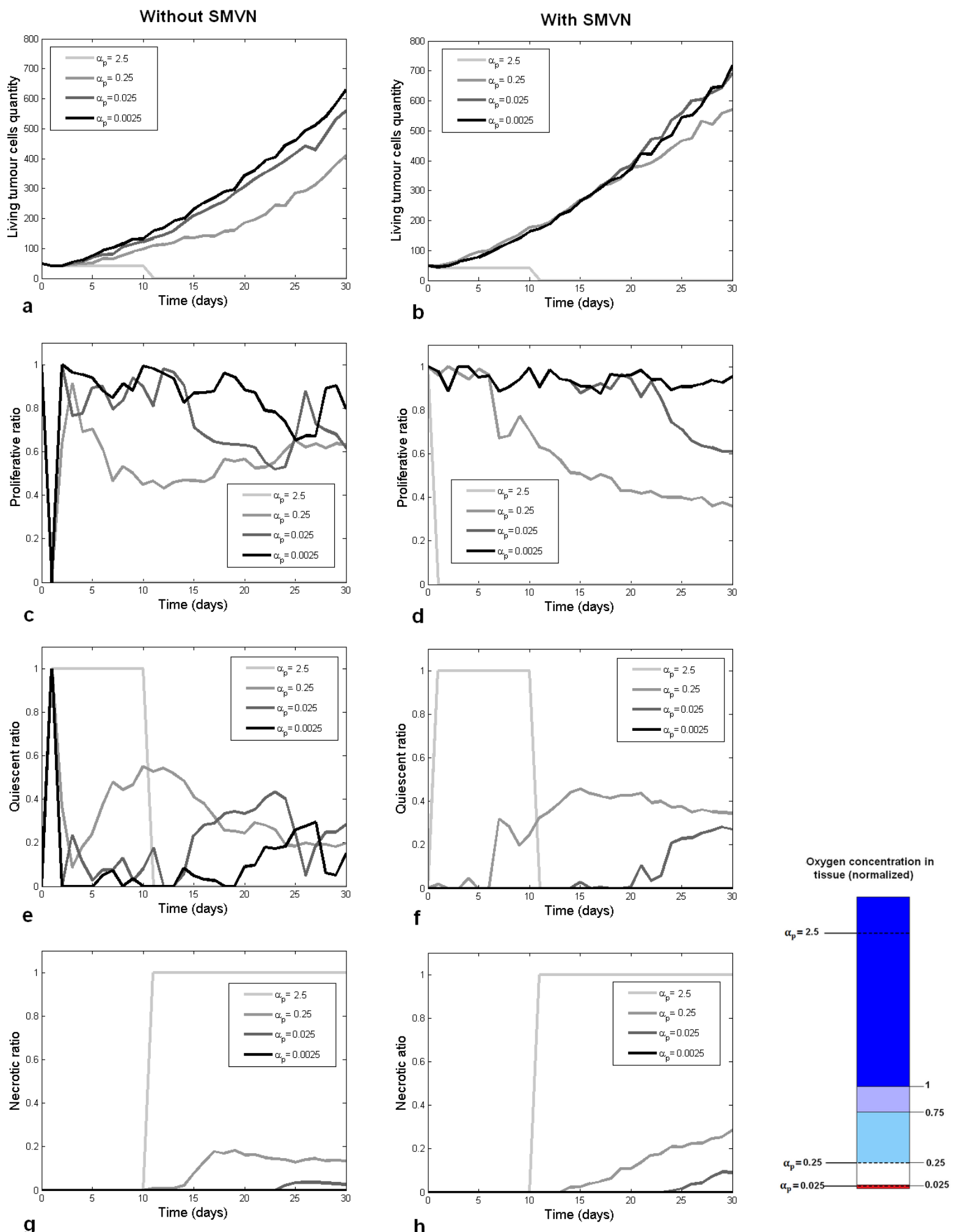

Figure 12: 
Figure 13
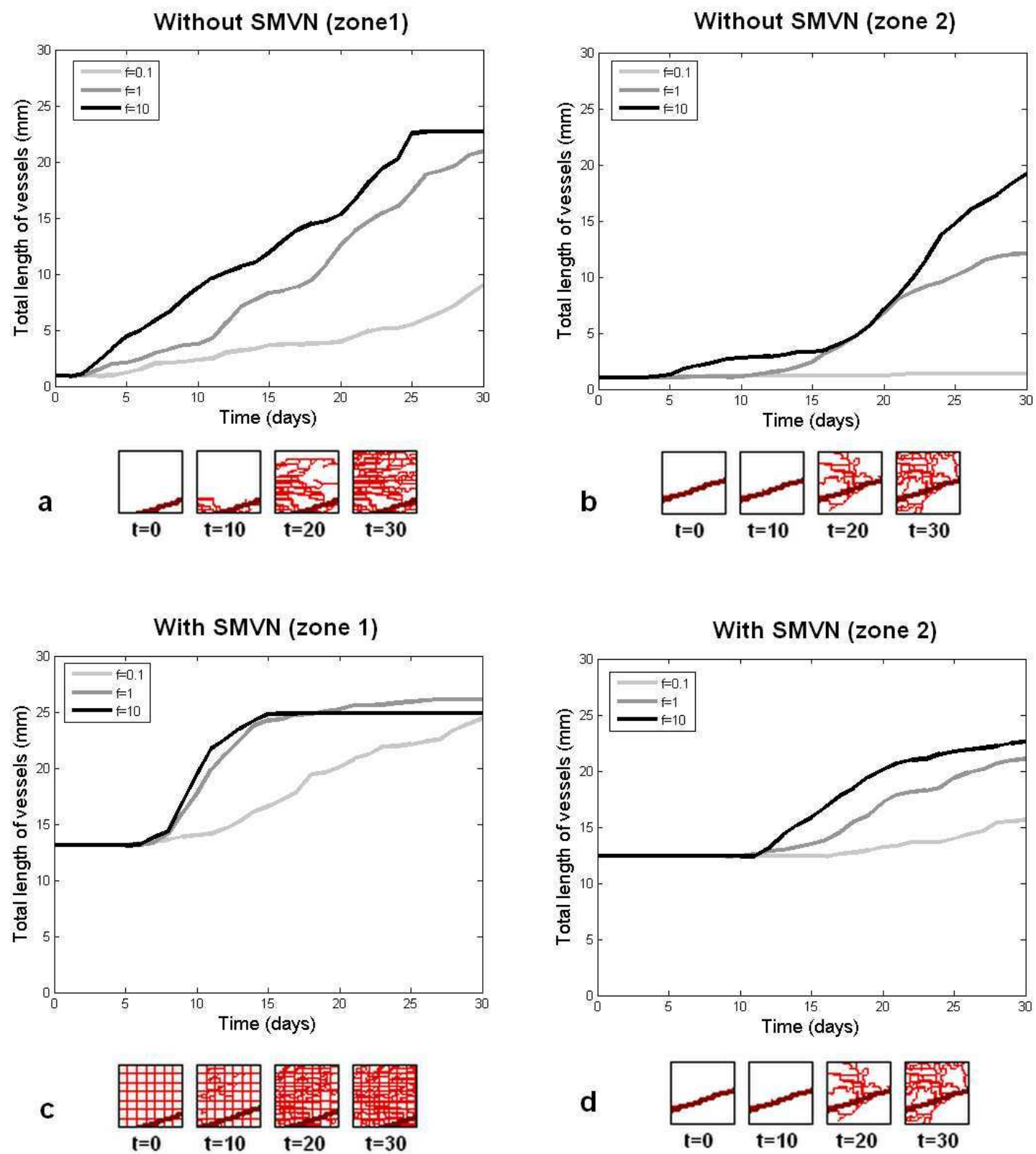

Figure 13: 
Figure 14
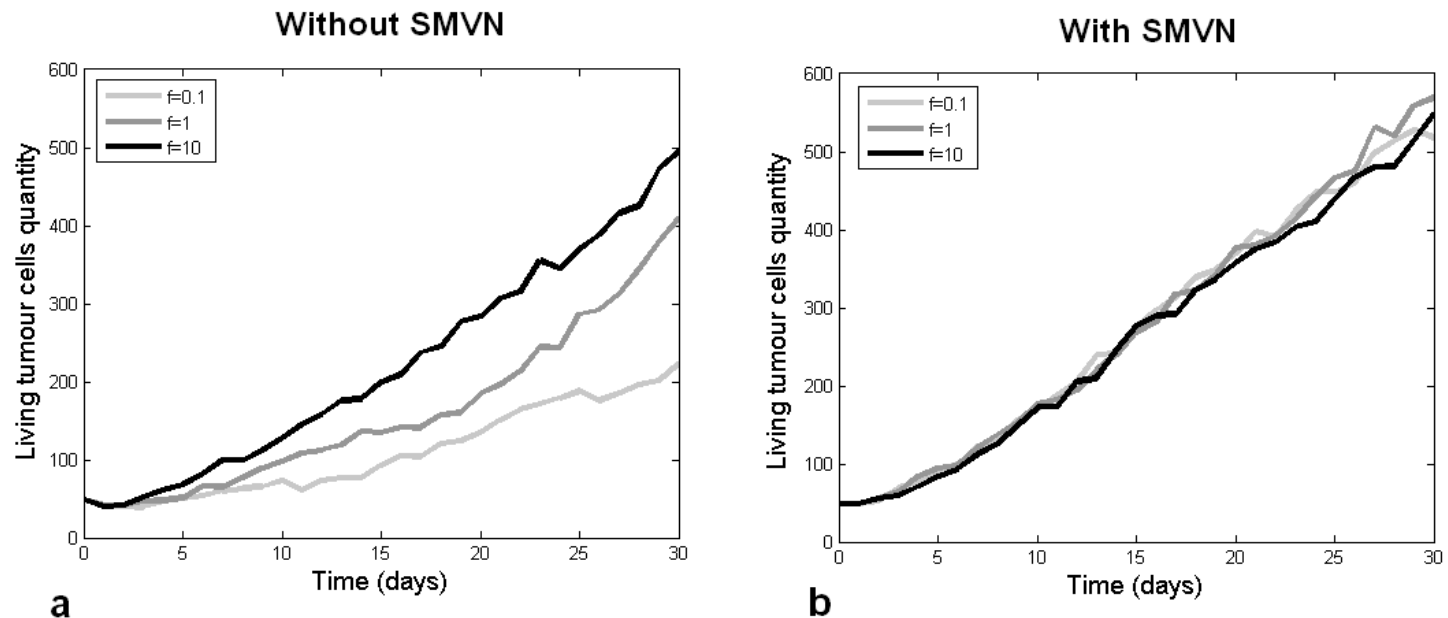

Figure 14: 
Figure 15

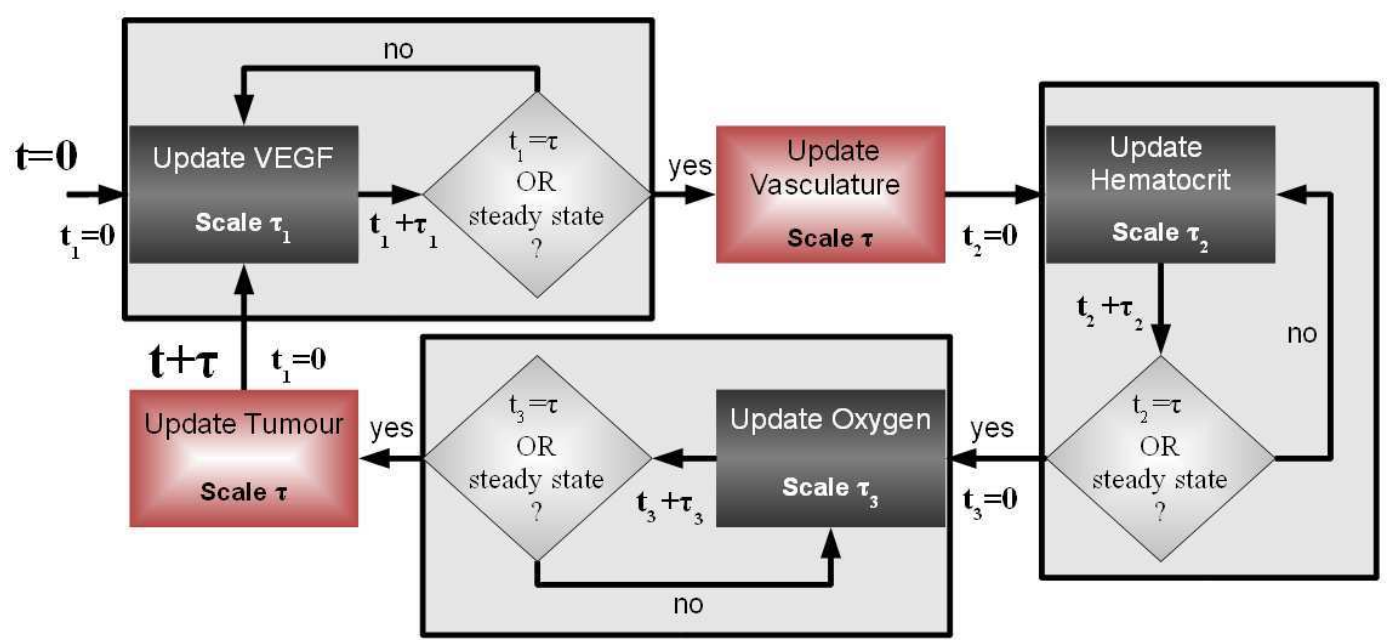

Figure 15: 


\section{Appendix}

\section{Appendix A. Simulation parameters}

All through the simulations, the space step used is $\Delta=\Delta_{x}=\Delta_{y}=25 \mu \mathrm{m}$, corresponding to the space occupied by one cell in the model. However, the model is multiscale regarding the time step, which adapts to update the different elements during the simulation, as shown in figure 15.

The main time step of the model $\tau$ is equal to 0.01 days. It is the time step used to update the tumour evolution and the microvascular network along with the matrix degrading enzyme and the fibronectin degradation. The model integrates three processes with smaller timesteps for VEGF and oxygen diffusion, and for the blood flow which concerns the update of the hematocrit. All these integrated processes are stopped when a steady state is reached or when the elapsed time for the considered update is about to overtake the main time step $\tau$ (Fig. 15).

All diffusion equations are solved with the ADI method and Neumann boundary conditions. In order to be more accurate to represent VEGF and oxygen diffusion, the timesteps used in the associated processes are respectively fixed to $\tau_{1}=\Delta^{2} / D_{V} \approx 22 \mathrm{~s}$ and $\tau_{3}=\Delta^{2} / D_{O_{2}} \approx 0.26 \mathrm{~s}$. For each time step $\tau_{2}$, the hematocrit is only updated in the vessel sections where hematocrit inflow and outflow are different. The value of this time step vary over the simulation. It is set to the time which is necessary to fill the vessel section which has the highest blood flow speed. 


\section{Table Legends}

Table 1: Parameter values.

Table 2: Vessels sprouting ability depending on VEGF concentration.

\section{Figure Legends}

Figure 1: Microscopic images of the vascular evolution resulting from the introduction of a piece of tumour (glioma cells). On the initial day (a), the piece of tumour is introduced under the glass of the skinfold chamber. Three days later (b) alterations in the vascular network are clearly visible with an increased density of small vessels around the tumour mass and the dilation of most of the vessels of the initial network.

Figure 2: Extraction of the skeleton of the vascular network in 3 steps. (1) A square window of interest is selected from image (side length of the square is $5.5 \mathrm{~mm}$ ); (2) the image is inverted and a brightness threshold is applied; (3) the nodes of the vascular skeleton are detected and are used to define each vessel segment $s_{i}$ characterized by their length $L_{i}$, diameter $D_{i}$ and inclination from horizontal $I_{i}$. The reference point for segment numbering is arbitrarily defined (big grey bullet). We note that the stain indicated by the white arrow is due to local bleeding which occurred when the dorsal window was implanted. It then quickly disappeared (see figure 1 which shows a later stage of the same vascular network when the tumour is introduced).

Figure 3: Relationship between the main modules and elements of the computational model.

Figure 4: Simulation domain for the cellular automaton. Tumour cells occupy elements of the grid $(e)$ and the vessels occupy the edges of this grid defined between two nodes $(N)$. 
Figure 5: Flowchart for tumour cells evolution. A timer is assigned to each cell to measure the time spent in a given state. $t_{P}$ is the timer assigned to proliferative cells. It is set to $t_{P}=t_{0}=0$ for the two daughter cells right after division of the mother cell occurring at the end of the $M$-phase when $t_{P}=t_{4}$ (mitosis). In normal oxygen condition, this timer is incremented with time step $\Delta t$. When a cell enters hypoxia, that is the $G_{0}$-phase, its timer $t_{P}$ is stopped and switched to $t_{Q}=0$. As long as the cell remains quiescent, its timer $t_{Q}$ is incremented by $\Delta t$. If oxygen becomes available again, the cell returns to the proliferation state and its timer is switched back to its value $t_{P}$ at which is was stopped. If $t_{Q} \geq T_{\text {limit }}$ then the cell becomes necrotic and switches irreversibly to timer $t_{N}$ which runs to infinity. The cell can also die by apoptosis (programmed cell death). It happens in this automaton if space is lacking for cell division. In that case, the cell timer is switched also irreversibly to $t_{A}$. It is incremented by $\Delta t$ until it reaches its limit $t_{A}=t_{\text {apop }}$ above which the cell is killed and is cleaned from the simulation space. All parameters are given in Table 1 in appendix Appendix A.

Figure 6: Visualization of the skin vasculature of a mouse at different scales. The picture on the left shows a macroscopic view of the vasculature (size of the window frame is $1 \mathrm{~cm}$ ). A region is selected in this picture and is shown at a higher resolution (middle picture). Capillaries of the SMVN can be seen. Three zones are selected and the capillaries are highlighted in each zone in the right panel. The average distance between the capillaries is around $100 \mu \mathrm{m}$.

Figure 7: Simulation results of the tumour development in the dorsal chamber for the tissue case without SMVN. The tumour evolution is followed over 70 days. Top to bottom: vascular network and tumour, oxygen concentration, degradation of matrix fibres, VEGF concentration, corresponding experimental vascular network (first picture of last line).

Figure 8: Simulation results of the tumour development in the dorsal chamber for the tissue case with SMVN. The tumour evolution is followed over 70 days. Top to bottom: vascular network and tumour, oxygen concentration, degradation of matrix fibres, VEGF concentration, corresponding experimental vascular network (first picture of last line). Two small zones are defined in the first picture to follow the evolution of the vascular density. From left to right: zone1 and zone2.

Figure 9: Evolutions of the tumour cell quantity (a) and of the tumour radius (b) over time. 
Figure 10: Cell quantity evolution with time for each tumour cell's state without (a) and with (b) SMVN.

Figure 11: Evolutions with time of the quiescent cells quantity (a), VEGF quantity (b), VEGF quantity per day (c) and maximum VEGF concentration in the tissue.

Figure 12: Evolution with time when varying the oxygen threshold $\alpha_{p}$ of the living tumour cells population (proliferative+quiescent cells) $(\mathrm{a}, \mathrm{b})$ as well as proliferative $(\mathrm{c}, \mathrm{d})$, quiescent $(\mathrm{e}, \mathrm{f})$ and necrotic ratio $(\mathrm{g}, \mathrm{h})$ without and with SMVN respectively. Oxygen thresholds between proliferation and quiescence $\left(\alpha_{p}\right)$. The scale is normalized from the maximum concentration value of oxygen measured in the tissue before angiogenesis starts.

Figure 13: Evolutions with time of the total vessel length in the zones defined in figure 8a. Case without SMVN in zone1 (a) and zone2 (b). Case with SMVN in zone1 (c) and zone2 (d).

Figure 14: Evolution with time of the living tumour cells population (proliferative+quiescent cells) without SMVN (a) and with SMVN (b) when varying the sprouting probability of the vascular network by a factor $f$.

Figure 15: Algorithm for the computational model. $\tau$ is the main time step used for the simulation and is related to the slower time-scale (scale of hours). $\tau_{1}, \tau_{2}$ and $\tau_{3}$ represent the intermediate time steps to update the processes which occur on fastest time scales (from milliseconds to seconds). 